ARTICLE

\title{
MAVS is energized by Mff which senses mitochondrial metabolism via AMPK for acute antiviral immunity
}

\author{
Yuki Hanada (1) 1,2, Naotada Ishihara (1) 1,3凶${ }^{\text {, Lixiang Wang (iD) }}{ }^{2,4}$, Hidenori Otera ${ }^{5}$, Takaya Ishihara ${ }^{1}$,
} Takumi Koshiba (10) ${ }^{6}$, Katsuyoshi Mihara ${ }^{5}$, Yoshihiro Ogawa (i] ${ }^{2}$ \& Masatoshi Nomura (1) ${ }^{2,7}$

Mitochondria are multifunctional organelles that produce energy and are critical for various signaling pathways. Mitochondrial antiviral signaling (MAVS) is a mitochondrial outer membrane protein essential for the anti-RNA viral immune response, which is regulated by mitochondrial dynamics and energetics; however, the molecular link between mitochondrial metabolism and immunity is unclear. Here we show in cultured mammalian cells that MAVS is activated by mitochondrial fission factor (Mff), which senses mitochondrial energy status. Mff mediates the formation of active MAVS clusters on mitochondria, independent of mitochondrial fission and dynamin-related protein 1. Under mitochondrial dysfunction, Mff is phosphorylated by the cellular energy sensor AMP-activated protein kinase (AMPK), leading to the disorganization of MAVS clusters and repression of the acute antiviral response. Mff also contributes to immune tolerance during chronic infection by disrupting the mitochondrial MAVS clusters. Taken together, Mff has a critical function in MAVS-mediated innate immunity, by sensing mitochondrial energy metabolism via AMPK signaling.

\footnotetext{
${ }^{1}$ Department of Biological Sciences, Graduate School of Science, Osaka University, 1-1 Machikaneyama, Toyonaka 560-0043, Japan. ${ }^{2}$ Department of Medicine and Bioregulatory Science, Graduate School of Medical Science, Kyushu University, 3-1-1 Maidashi, Higashi-ku, Fukuoka 812-8582, Japan.

${ }^{3}$ Department of Protein Biochemistry, Institute of Life Science, Kurume University, 67 Asahi-machi, Kurume 830-0011, Japan. ${ }^{4}$ Department of Medical Biochemistry, Kurume University School of Medicine, 67 Asahi-machi, Kurume 830-0011, Japan. ${ }^{5}$ Department of Molecular Biology, Graduate School of Medical Science, Kyushu University, 3-1-1 Maidashi, Higashi-ku, Fukuoka 812-8582, Japan. ${ }^{6}$ Department of Chemistry, Faculty of Science, Fukuoka University, 8-19-1 Nanakuma, Jonan-ku, Fukuoka 814-0180, Japan. ${ }^{7}$ Department of Endocrinology and Metabolism, Kurume University School of Medicine, 67 Asahi-machi, Kurume 830-0011, Japan. ${ }^{凶}$ email: naotada@bio.sci.osaka-u.ac.jp
} 
$\mathrm{M}$ itochondria are multifunctional organelles that have critical roles not only in energy production by oxidative phosphorylation but also in various cellular signaling pathways such as apoptosis, calcium signaling, oxidative stress, and innate immunity ${ }^{1-3}$. Mitochondria dynamically change their morphology by a balance between active fusion and fission in response to intracellular signaling and the surrounding environment ${ }^{4,5}$. In mammals, mitochondrial membrane fusion is regulated by GTPase proteins: two mitofusin isoforms (Mfn1 and Mfn2) on the outer membrane (OM), and optic atrophy type 1 (OPA1) on the inner membrane ${ }^{5}$. Mitochondrial fission is also driven by another GTPase protein, dynamin-related protein 1 (Drp1). Drp1 is localized primarily in the cytosol, is targeted to mitochondrial fission sites on the OM, and mediates mitochondrial fission ${ }^{5}$. Mitochondrial fission has been implicated in various cellular signaling pathways and diseases, such as apoptosis ${ }^{4}$, embryonic development ${ }^{6,7}$, neurodegeneration ${ }^{6-8}$, mitophagy ${ }^{5}$, metabolic disorders ${ }^{9,10}$, and immunity ${ }^{11}$. Several Drp1 receptors have been identified in mammals, such as mitochondrial fission factor (Mff) ${ }^{12,13}$ and mitochondrial dynamic protein of 49 and $51 \mathrm{kDa}$ (MiD49 and MiD51, respectively) ${ }^{14-16}$. Mff is an OManchored protein containing a putative Drp1-binding region in its $\mathrm{N}$-terminus ${ }^{12,13}$. Mutations in Mff cause neurodegenerative disease in humans ${ }^{17-19}$, and gene trap disruption of Mff in mice leads to heart failure, infertility, neuromuscular disorders, and growth abnormalities in several tissues ${ }^{20}$; however, the details of the physiological roles of Mff remain unknown.

RNA virus infection induces the innate immune response, concomitant with mitochondrial morphological changes ${ }^{1}$. Mitochondrial antiviral signaling (MAVS) protein, also known as IPS$1 / \mathrm{VISA} / \mathrm{Cardif}$, plays a central role in the induction of the antiviral innate immune response on mitochondria ${ }^{21-24}$. A Cterminal transmembrane domain of MAVS for anchoring to the mitochondrial $\mathrm{OM}$ is required for its antiviral activity ${ }^{22,23}$. MAVS is an adaptor of retinoic acid-inducible gene-I-like receptors (RLRs) such as RIG-I and MDA5, which are RNA helicases that recognize intracellular viral double-stranded RNA (dsRNA) ${ }^{1}$. MAVS has an N-terminal caspase-recruitment domain (CARD) that interacts with the CARDs of RLRs ${ }^{21-24}$. Once MAVS is activated via its interaction with RLRs, it forms a highly oligomeric comple ${ }^{25}$ and provides a platform for recruiting downstream signal transducers and kinases, leading to the robust induction of type 1 interferon (IFN) proteins and other inflammatory cytokines $1,21-24$. Several reports have shown that mitochondrial dynamics are related to the MAVSmediated antiviral response ${ }^{26-29}$. Mfn2 interacts with MAVS and inhibits the antiviral response ${ }^{26}$; however, knockout (KO) of both Mfn isoforms severely arrests MAVS signaling ${ }^{30}$. Meanwhile, treatment with respiratory inhibitors, the increased expression of uncoupling protein 2, or mutations in mitochondrial DNA (mtDNA) also lead to the arrest of MAVS signaling ${ }^{30,31}$. However, the molecular mechanism by which mitochondria regulate the antiviral response and the reason why the immune response occurs on mitochondria remain largely unknown.

Mitochondrial dynamics and signaling are linked to energy homeostasis and metabolism ${ }^{32,33}$. Under respiratory dysfunction, the transmembrane domain of long-form OPAl is processed to generate short-form OPA1, leading to mitochondrial fragmentation via the attenuation of mitochondrial fusion ${ }^{34}$. In contrast, under energy starvation, Drp1 is phosphorylated, resulting in mitochondrial elongation as a mechanism to escape from mitophagy 35,36 . Recent studies have demonstrated that Mff is phosphorylated by AMP-activated protein kinase (AMPK), leading to the activation of mitochondrial fission ${ }^{37,38}$. Immune responses are known to be susceptible to energy status, as suggested by in vivo analysis of mice ${ }^{39,40}$ and cell-based analysis ${ }^{41}$; however, the molecular mechanism for the energetic regulation of innate immune signaling has not been characterized in depth.

Here we show an important function for Mff in the regulation of MAVS. Mff mediates MAVS cluster formation on the mitochondrial OM to activate the innate immune response, in a membrane fission-independent manner. The dual functions of Mff in mitochondrial fission and MAVS activity are both regulated by AMPK in response to energetic conditions. In addition, after acute activation, MAVS clusters are lost from the mitochondria, switching off the antiviral response. Thus Mff switches from a strong and transient immune response under energy-rich conditions to a weak and continuous immune response under energy-starved conditions. Taken together, our results provide evidence for a missing link between bioenergetics and the innate immune response on mitochondria.

\section{Results}

Mff is required for the MAVS-mediated antiviral response. Mff is a mitochondrial OM protein that mediates the recruitment of the mitochondrial fission-stimulating GTPase Drp1 to mitochondrial fission sites $^{5}$, which is enhanced by AMPK-dependent phosphorylation under respiratory dysfunction ${ }^{38}$. To investigate the roles of Mff, we initially established Mff $\mathrm{KO}$ mouse embryonic fibroblasts (MEFs; Supplementary Fig. 1a-d). As expected, Mff KO MEFs had elongated mitochondria (Supplementary Fig. 1c) and failed to recruit Drp1 to the mitochondria (Supplementary Fig. 1d). Next, we examined the role of Mff in MAVS-mediated antiviral innate immunity. Type I IFN family proteins, including several isoforms of IFN- $\alpha$ and the single isoform of IFN- $\beta$, are antiviral cytokines that are induced to inhibit viral replication ${ }^{42}$. After introducing the low molecular weight (LMW) form of poly (I:C), a viral dsRNA analog, the secretion of IFN- $\beta$ and interleukin 6 (IL-6) analyzed by enzyme-linked immunosorbent assay (ELISA) was suppressed severely in Mff KO MEFs (Fig. 1a, b, "poly(I:C)-LMW"), suggesting that Mff plays a critical role in the MAVS-mediated antiviral response. Mitochondrial morphological changes after the viral RNA introduction $27,29,43,44$ were severely repressed in Mff KO MEFs and in Drp1 KO MEFs (Supplementary Fig. 1e). Drp1 recruitment was also severely repressed in Mff KO MEFs (Supplementary Fig. 1f). The immediate fragmentation of mitochondria in wild-type (WT) MEFs was subsequently recovered to normal mitochondrial morphology toward elongation under the antiviral response (Supplementary Fig. 1e), as reported ${ }^{27}$. These data suggested that Mff is required for cytokine induction and Drp1-dependent mitochondrial fission during the antiviral response.

MAVS mediates signal transduction from RNA sensors to kinases and transcription factors, such as TANK-binding kinase 1 (TBK1) and interferon regulatory factor 3 (IRF3), for IFN- $\beta$ induction ${ }^{1}$. When we introduced various concentrations of poly (I:C)-LMW, the antiviral response occurred in a poly(I:C) concentration-dependent manner, and the response was severely repressed in the absence of Mff (Supplementary Fig. 2a, b). When we tested other pathogens (Fig. 1c), high molecular weight (HMW) form of poly(I:C)-induced cytokine secretion was also suppressed in Mff KO cells (Fig. la, b). Unexpectedly, the introduction of poly(dI:dC), an analog of cytosolic viral dsDNA, also induced cytokine secretion in an Mff-dependent manner (Fig. 1a, b), although the intracellular DNA only weakly induced transcription of these cytokines (Supplementary Fig. 2a, b). It is possible that the MAVS pathway could be activated by cross-talk with the cGAS/STING pathway, known to be activated by viral DNAs (Fig. 1c), as previously reported ${ }^{27,45,46}$, although the 
a

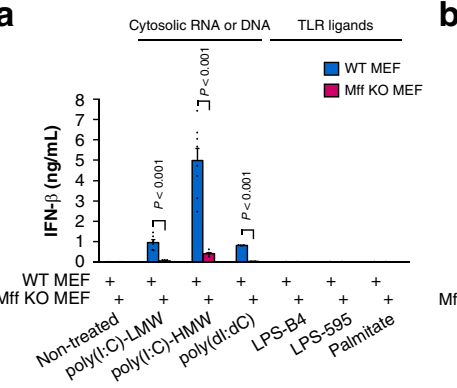

d

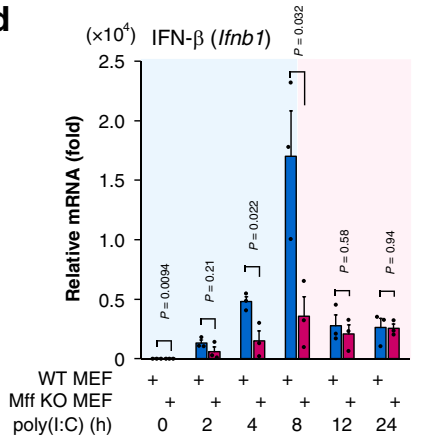

g

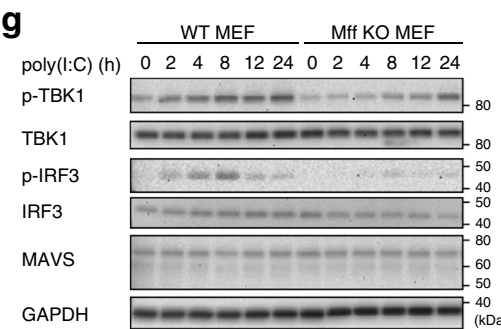

h

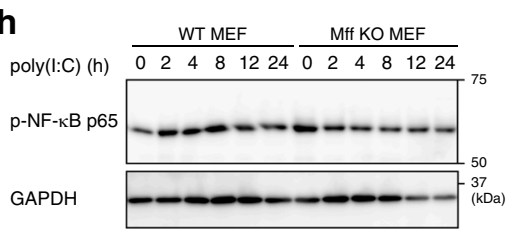

k

K IFN- $\beta($ IFNB1)

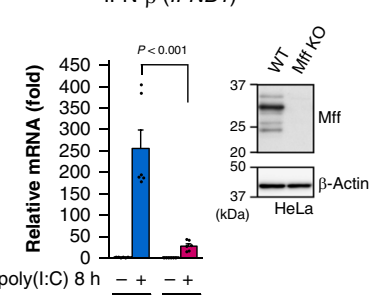

HeLa $\frac{-+}{\text { WT }} \frac{-+}{\text { Mff KO }}$

n

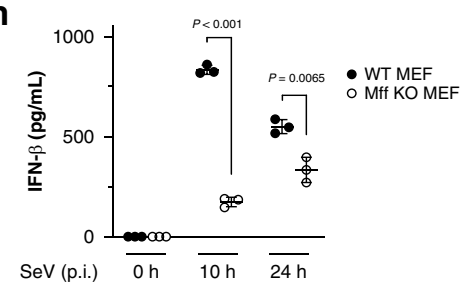

molecular details remain to be determined. In contrast, the production of IL-6 via Toll-like receptor 4 (TLR4), which is independent of MAVS ${ }^{24}$ and can be stimulated by TLR4 ligands such as lipopolysaccharide (LPS) and palmitate ${ }^{47}$, was comparable between WT and Mff KO MEFs (Fig. 1b). These results suggested that Mff specifically modulates the MAVS-mediated antiviral response (Fig. 1c).
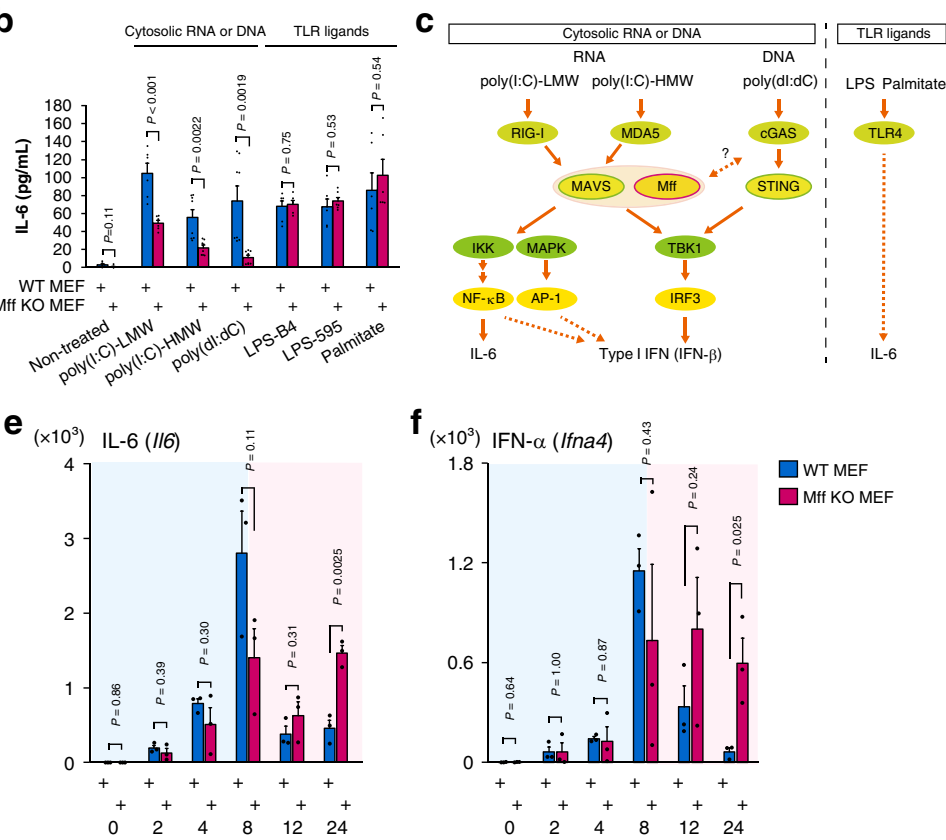

$\mathbf{f}_{\left(\times 10^{3}\right) \text { IFN- } \alpha(\text { Ifna4 })}$
1.8
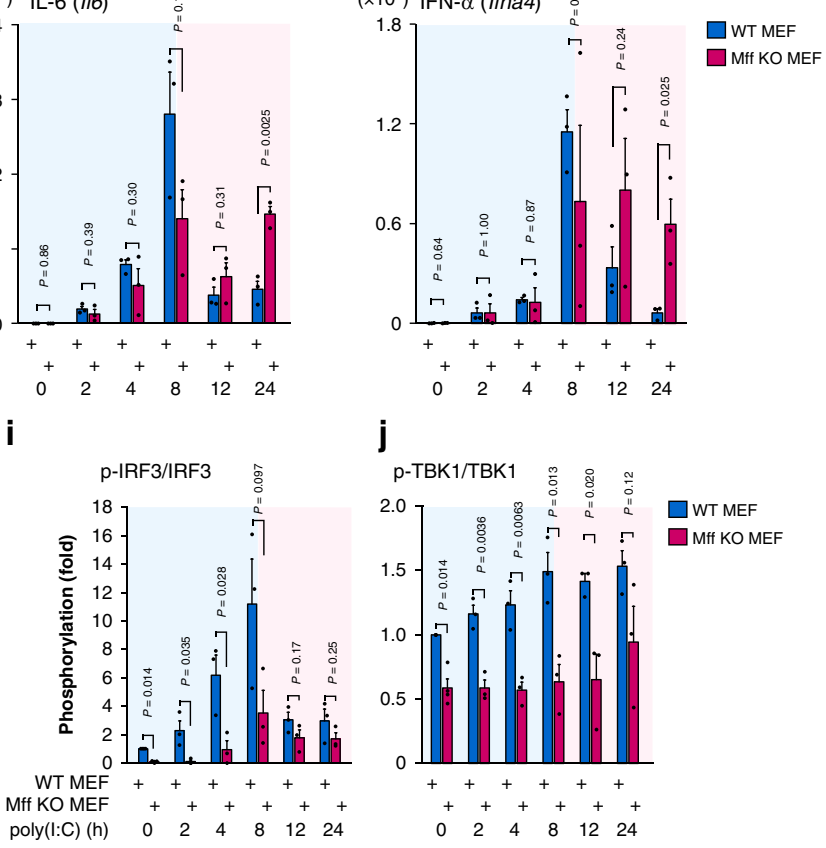

m
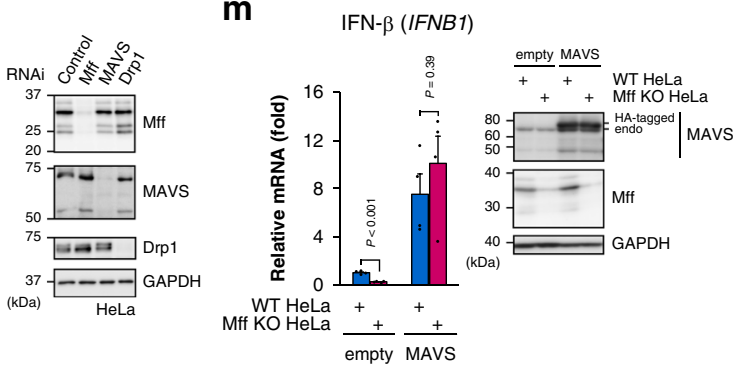

Next we analyzed the time profile of the MAVS-mediated antiviral response. When we examined the mRNA expressions of antiviral cytokines in WT MEFs, IFN- $\beta$ (Fig. 1d), IL-6 (Fig. 1e), and IFN- $\alpha$ (Fig. 1f) were induced until $8 \mathrm{~h}$ after poly(I:C) transfection (acute phase), and their expression was drastically suppressed after $8 \mathrm{~h}$ (tolerance phase). A similar temporal response was observed for the level of phosphorylation of IRF3 
Fig. 1 Mff is required for MAVS-mediated antiviral signaling. a, b IFN- $\beta$ (a) and IL-6 (b) secretion in WT and Mff KO MEFs. Cells were transfected with poly(I:C)-LMW (WT, KO: $n=6,5$ in $\mathbf{a} ; n=6$ in $\mathbf{b})$, poly(I:C)-HMW ( $n=8)$, or poly(dl:dC) (WT, KO: $n=4,5$ in $\mathbf{a}$; $n=8$ in $\mathbf{b})$, or cultured with LPS or palmitate $(n=6)$ for $8 \mathrm{~h}$. $P$ values; two-tailed unpaired $t$ test. c Scheme of the innate immune cascades examined in this study. Defects of innate immune response were observed around MAVS under Mff deficiency. d-f qRT-PCR analyses of IFN- $\beta$ (d), IL-6 (e), and IFN- $\alpha$ (f) mRNA in WT and Mff KO MEFs transfected with poly $(\mathrm{I}: \mathrm{C})$-LMW $(1 \mu \mathrm{g} / \mathrm{mL})$ for the indicated times. $P$ values; two-tailed unpaired $t$ test $(n=3)$. The blue shaded parts of the graphs $(0-8 \mathrm{~h})$ represent the acute phase after poly $(\mathrm{I}: \mathrm{C})$, and the red shaded parts $(8-24 \mathrm{~h})$ represent the tolerant phase. $\mathbf{g}$-j Immunoblot analyses of IRF3, TBK1 (g) and NF-KB (h) phosphorylation as in $\mathbf{d - f}$. Phosphorylation levels of IRF3 and TBK1 were normalized to its total protein levels. $P$ values; two-tailed unpaired $t$ test $(2-24 h ; n=3)$ or Mann-Whitney's $U$ test $(0$ h; $n=4)$. $\mathbf{k}$, I qRT-PCR analysis of IFN- $\beta$ mRNA in WT and Mff KO HeLa cells (k) or in HeLa cells transfected with control, Mff, MAVS, or Drp1 siRNA (I). Cells were transfected with poly (I:C)-LMW $(1 \mu \mathrm{g} / \mathrm{mL})$ for the indicated times. The protein levels of Mff and other RNAi genes were analyzed by immunoblotting. $P$ values; two-tailed unpaired $t$ test $(\mathbf{k}, n=6$; I, Control, Mff, MAVS, Drp1: $n=6,6,3,3)$. m qRT-PCR analysis of IFN- $\beta$ mRNA in WT HeLa and Mff KO HeLa cells transfected with empty vector or HA-tagged MAVS. $P$ values; two-tailed unpaired $t$ test ( $n=$ 4). Overexpressed MAVS protein was detected by immunoblotting. $\mathbf{n}$ IFN- $\beta$ secretion in WT and Mff KO MEFs infected with Sendai virus (SeV) at 10 and $24 \mathrm{~h}$ post infection (p.i.). $P$ values; two-tailed unpaired $t$ test $(n=3)$. Data represent means \pm SEM from three independent experiments $(\mathbf{a}, \mathbf{b}, \mathbf{d}-\mathbf{f}, \mathbf{i}, \mathbf{j}-\mathbf{m})$ or representative one experiment (n) with similar results. Immunoblot analyses were repeated independently three times with similar results. Source data are provided as a Source data file.

in WT MEFs analyzed by immunoblotting (Fig. 1g, i). In contrast, the acute antiviral response for cytokine mRNA induction (Fig. 1d-f) and IRF3 phosphorylation (Fig. 1g, i) was suppressed in Mff KO MEFs. The phosphorylation of TBK1 (Fig. 1g, j) and activation of nuclear factor- $\kappa \mathrm{B}$ (Fig. 1h, "p-NF- $\mathrm{B}$ p 65 ") modestly induced in WT MEFs was also repressed in Mff KO MEFs. These results suggest that Mff regulates signal transduction of the MAVS-mediated antiviral response.

To examine the involvement of Mff in the antiviral response in human cells, we analyzed HeLa cells and found the impaired induction of IFN- $\beta$ mRNA in Mff KO HeLa cells ${ }^{16}$ (Fig. 1k) and in Mff- or MAVS-repressed HeLa cells by RNA interference (RNAi; Fig. 11). In contrast, the antiviral cytokine IFN- $\beta$ was induced normally in Drp1-RNAi HeLa cells, as in control cells (Fig. 11), suggesting that mitochondrial fission is not essential for the antiviral response, consistent with previous reports of Drp1 depletion or the expression of a dominant-negative mutant Drp1 $\mathrm{K}_{38} \mathrm{~A}^{27,48}$. It was also reported that the MAVS overexpression causes the induction of IFN proteins without virus infection, possibly by auto-activation ${ }^{21-24}$. When MAVS was overexpressed in Mff KO HeLa cells, IFN- $\beta$ was highly induced, as observed in WT HeLa cells (Fig. 1m), suggesting that Mff acts upstream of MAVS activation and the subsequent induction of type I IFN. Taken together, we found that Mff selectively affects the MAVSmediated antiviral signaling pathway.

We further examined the function of Mff during RNA virus infection. At $10 \mathrm{~h}$ after Sendai virus (SeV) infection, IFN- $\beta$ secretion observed in WT MEFs was severely repressed in Mff KO MEFs (Fig. 1n). However, the antiviral response in Mff KO cells was prolonged for $24 \mathrm{~h}$ after RNA virus infection, although the antiviral response was exhausted in WT cells (Fig. 1n). These results confirmed that Mff regulates MAVS-mediated anti-RNA viral response in a time-dependent manner.

Mff mediates MAVS cluster formation on mitochondria. Under antiviral signaling, MAVS forms huge oligomeric complexes on mitochondria ${ }^{25}$. When we visualized endogenously expressed MAVS in MEFs by immunofluorescence confocal microscopy, MAVS formed many accumulated dot-like clusters on mitochondrial tubules (Fig. $2 \mathrm{a}-\mathrm{c}$ ), consistent with previous observations under super-resolution microscopy ${ }^{43}$. To examine the role of Mff in the distribution of MAVS, we analyzed Mff KO MEFs and found a more diffuse distribution of MAVS on mitochondrial tubules (Fig. 2a, b), as well as the cytoplasmic distribution of Drp1 (Fig. 2c and Supplementary Fig. 1d). Then we measured the co-localization efficiency between MAVS and mitochondria. Because dispersal of MAVS clusters causes MAVS to have a more uniform distribution on mitochondria, this can be measured as an increase in the correlational coefficient between MAVS and mitochondrial signals. The quantification confirmed that Mff mediates the formation of MAVS clusters, which accumulated on a part of the mitochondria (Fig. 2d). Under double staining of MAVS and Mff, Mff partly but clearly overlapped with the MAVS clusters in WT MEFs and Drp1 KO MEFs (Fig. 2a, quantification in Fig. 2e). In contrast, the MAVS clusters rarely colocalized with Drp1 structures in WT MEFs (Fig. 2c, quantification in Fig. 2e). We further analyzed oligomerization of MAVS biochemically using clear-native polyacrylamide gel electrophoresis (CN-PAGE), showing that the formation of higherorder MAVS oligomers was repressed in Mff KO cells (Supplementary Fig. 2c). These data suggested that Mff regulates the mitochondrial distribution of MAVS, independently of Drp1dependent mitochondrial fission (model: Fig. 2f).

Mff regulates antiviral response independent of fission. As shown above, MAVS failed to form clusters and failed to mediate an efficient antiviral response in Mff-depleted cells. To further analyze the underlying molecular mechanism, we examined the domain structure of Mff protein required for the MAVSmediated antiviral response. We introduced three different FLAG-tagged Mff constructs into Mff KO MEFs (Fig. 3a): fulllength Mff ("Mff [WT]"); Mff lacking the C-terminal transmembrane domain ("Mff [ $\Delta \mathrm{C} 20]$ "), and Mff lacking 100 amino acid residues from the cytoplasmic N-terminal domain ("Mff $\left.[\Delta \mathrm{N} 100]^{\prime \prime}\right)^{13}$. After transient expression of these constructs in Mff KO MEFs, their distribution (Fig. 3b), immune response (Fig. 3c), and protein expression (Fig. 3d) were analyzed.

In Mff KO MEFs, Mff (WT) and Mff ( $\triangle$ N100) localized to the mitochondria, while Mff $(\Delta \mathrm{C} 20)$ was localized in the cytoplasm (Fig. 3b), because it did not have the transmembrane domain necessary for anchoring to the $\mathrm{OM}^{13}$. The highly elongated mitochondria in Mff KO MEFs were changed to fragmented mitochondria by Mff (WT) expression but not by Mff $(\triangle \mathrm{C} 20)$ or Mff ( $\triangle \mathrm{N} 100)$ expression (Fig. $3 \mathrm{~b}$ and Supplementary Fig. 3 ), and these two truncated mutants were unable to recruit Drp1 to the mitochondria (Supplementary Fig. 3a), as reported previously ${ }^{13}$. As expected, the antiviral response (Fig. 3c) and MAVS cluster formation (Fig. 3e, f) were rescued by Mff (WT) expression in Mff $\mathrm{KO}$ MEFs. In contrast, although Mff $(\Delta \mathrm{N} 100)$ was highly expressed (shown in the upper panel of Fig. 3d, immunoblot detected by an anti-FLAG antibody), Mff $(\Delta$ N100) expression failed to rescue the diffuse mitochondrial distribution of MAVS (Fig. 3e, f) and the defective antiviral signaling (Fig. 3c) in Mff KO MEFs, suggesting that the $\mathrm{N}$-terminal region of Mff is critical for mitochondrial fission and antiviral signaling. Next we analyzed Mff ( $\Delta$ C20); the protein expression level was lower than that of 
a
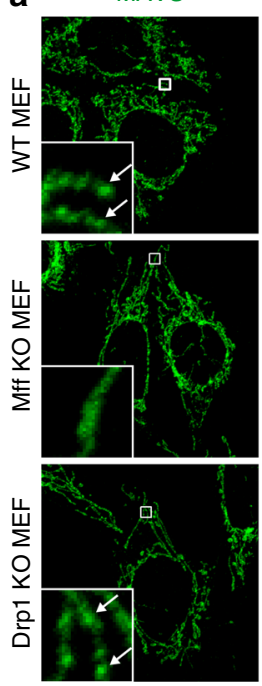

b
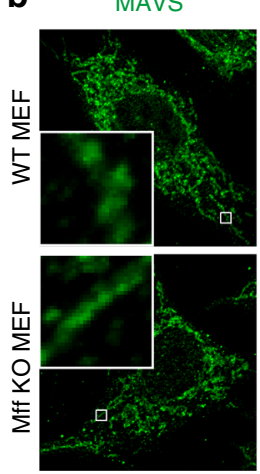

Mff
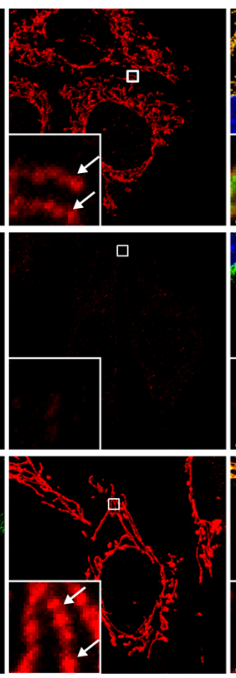

MitoTracker
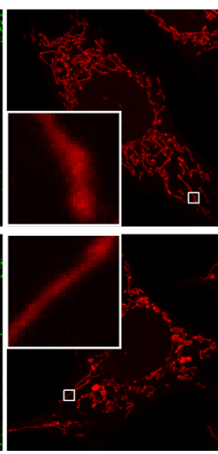

Nuclei/Merge
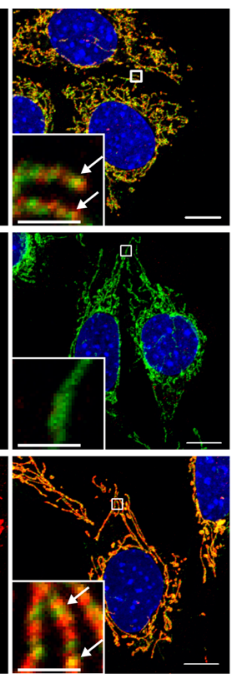

Nuclei/Merge
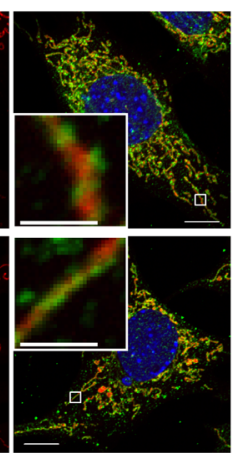

C
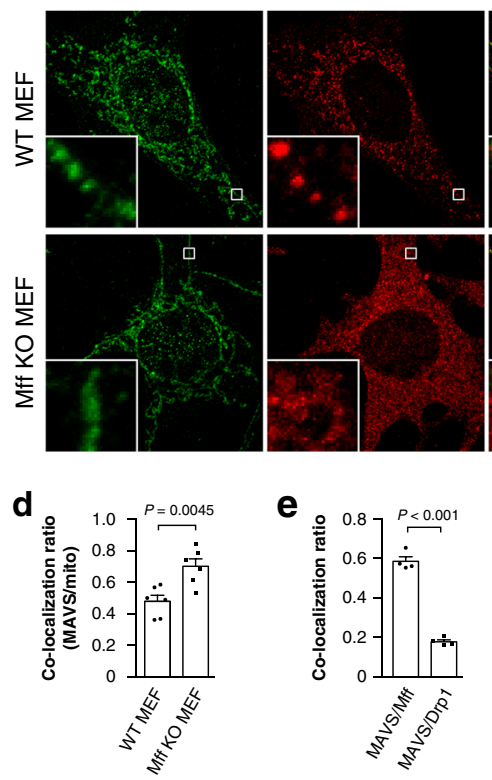

e

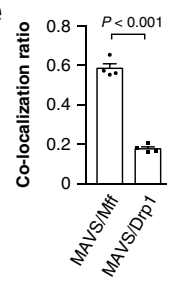

f

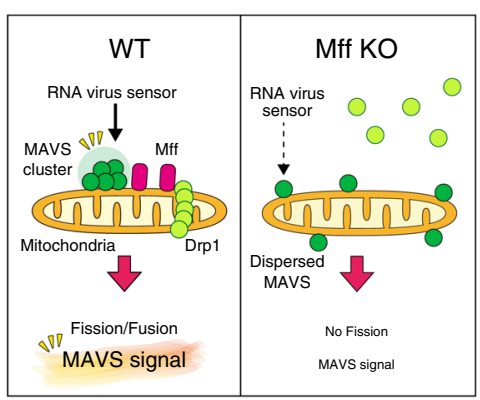

Fig. 2 Mff regulates accumulated distribution of MAVS on mitochondria. a Confocal microscopic images of MAVS and Mff. WT, Mff KO, and Drp1 KO MEFs were immunostained with anti-MAVS (green) and anti-Mff (red) antibodies. b Confocal microscopic images of MAVS and mitochondria. WT and Mff KO MEFs were stained with MitoTracker Red (mitochondria; red) and immunostained with an anti-MAVS antibody (green). c Confocal microscopic images of MAVS and Drp1. WT and Mff KO MEFs were immunostained with anti-MAVS (green) and anti-Drp1 (red) antibodies. Nuclei were stained with Hoechst 33342 (blue) in a-c. Scale bars: $10 \mu \mathrm{m}$ (whole) and $2 \mu \mathrm{m}$ (magnified) in a-c. d Co-localization ratio between MAVS and mitochondrial tubules (mitoRFP) in WT and Mff KO MEFs. P value; two-tailed unpaired $t$ test $(n=6)$. e Co-localization ratio between MAVS and Mff or Drp1 in WT MEFs. $P$ value; two-tailed unpaired $t$ test $(n=4)$. $\mathbf{f}$ Working hypothesis for the accumulated distribution of MAVS and the mitochondrial antiviral response regulated by Mff. Data represent means \pm SEM examined over more than three biologically independent samples (d, e). All microscopic analyses were repeated independently more than three times with similar results. Source data are provided as a Source data file.

Mff (WT) (Fig. 3d, detected by anti-FLAG). Unexpectedly, although Mff $(\Delta \mathrm{C} 20)$ lost the Drp1-dependent mitochondrial fission activity (Fig. 3b, e and Supplementary Fig. 3), Mff ( $\triangle \mathrm{C} 20)$ expression rescued the antiviral response (Fig. 3c) and MAVS cluster formation on mitochondria (Fig. 3e, f). Quantification of MAVS cluster formation on mitochondria as Fig. $2 \mathrm{~d}$ further confirmed that Mff $(\Delta \mathrm{C} 20)$ significantly rescued MAVS cluster formation (Fig. 3g). In conclusion, Mff has dual functions: it promotes mitochondrial fission via Drp1 recruitment to the mitochondria, and it mediates the antiviral response via MAVS cluster formation, which is independent of mitochondrial fission (model: Fig. 3h).

Mff phosphorylation by AMPK results in defective antiviral response. Mff is phosphorylated by AMPK under nutrientstarved conditions ${ }^{38}$; however, its physiological meaning is not understood. Here we examined the role of AMPK in the MAVSmediated antiviral response. Consistent with previous studies ${ }^{37,38}$, treatment with the AMPK activator AICAR increased the level of Mff phosphorylation, as well as the AMPKa phosphorylation (Fig. 4a). In the presence of AICAR, the induction of antiviral cytokines (IFN- $\beta$ and IL-6) was clearly repressed at the acute phase (before $8 \mathrm{~h}$ ), while the mild antiviral response was still maintained at the tolerant phase (after $8 \mathrm{~h}$ ) (Fig. $4 \mathrm{~b}, \mathrm{c}$ ), suggesting that the acute and temporal antiviral response is suppressed but prolonged by AMPK. In the absence of AICAR, phosphorylation of AMPKa and Mff was not significantly changed during the antiviral response in WT cells, suggesting that the poly(I:C) does not directly induce AMPK activation (Supplementary Fig. 2d). The mild and prolonged antiviral response observed after AICAR treatment (Fig. 4b, c) was similar to that seen in Mff $\mathrm{KO}$ cells (Fig. 1d, e). Under fluorescent microscopy, the accumulated mitochondrial distribution of MAVS became more diffuse after AICAR treatment (Fig. 4d), further suggesting that AMPK is involved in the distribution and activation of MAVS on mitochondria. We further analyzed the antiviral response in Mff KO MEFs and found that AICAR treatment until $8 \mathrm{~h}$ only weakly affected this response (Fig. 4e, f), suggesting that the inhibitory effect of AICAR on acute cytokine induction largely requires Mff. To examine further the role of Mff phosphorylation by AMPK, we constructed Mff proteins that were mutated at the S146 phosphorylation site and stably expressed them in Mff KO MEFs 
a

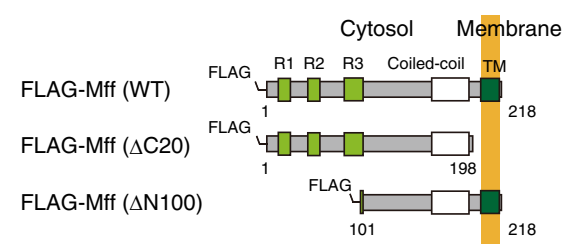

b FLAG-Mff MitoTracker Nuclei/Merge
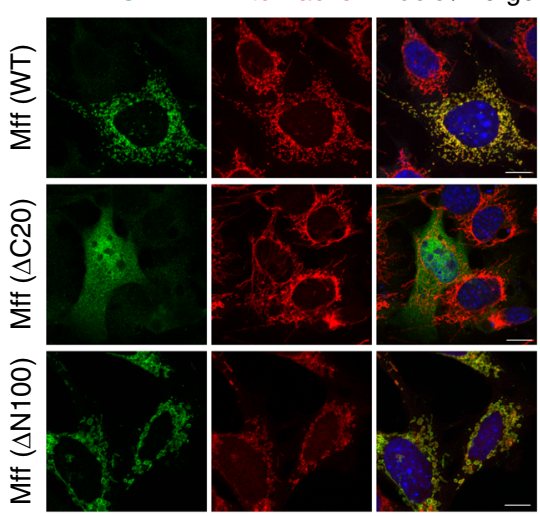

Mff KO MEF
C

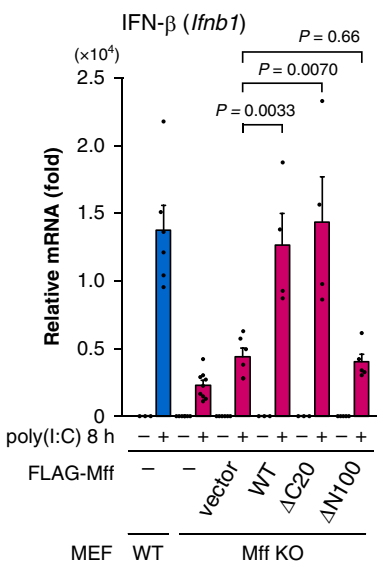

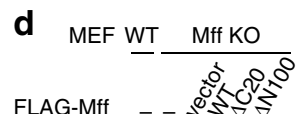

FLAG-Mff - -

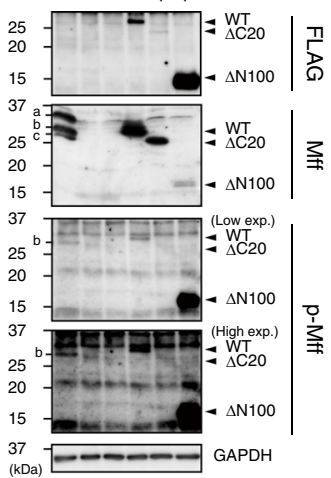

g

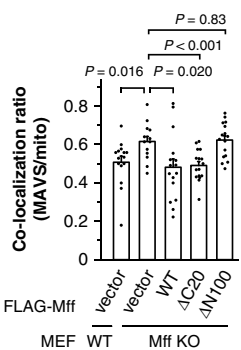

$\mathbf{e}$
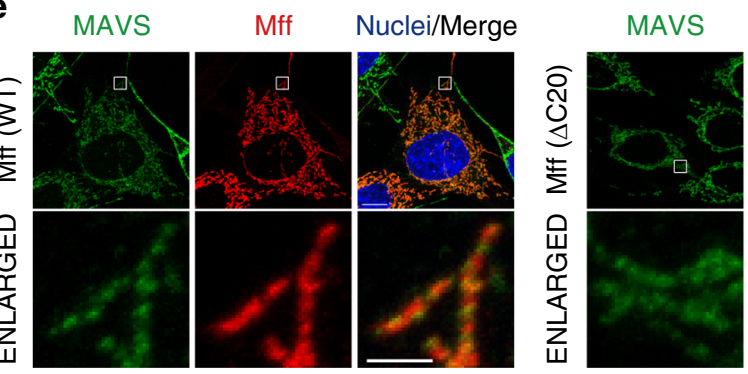

Mff

Nuclei/Merge

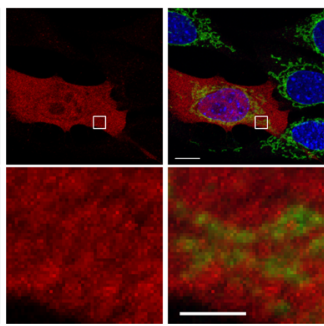

MAVS

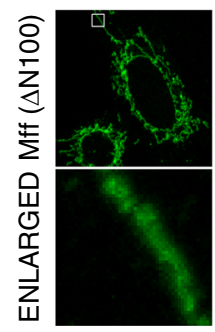

Mff

Nuclei/Merge
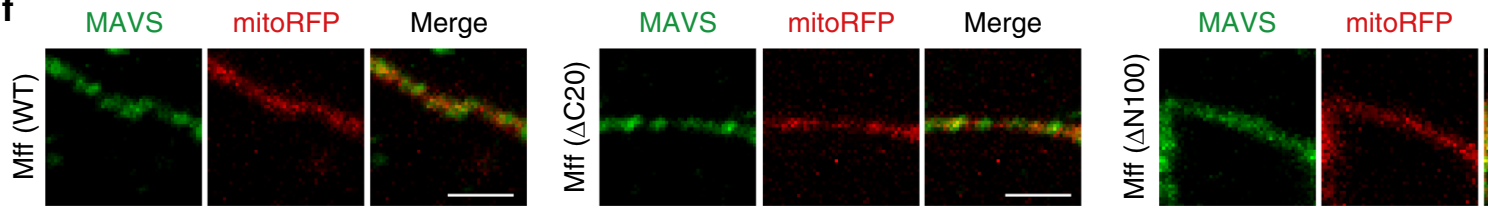

Merge

h $M$ Mff (WT)
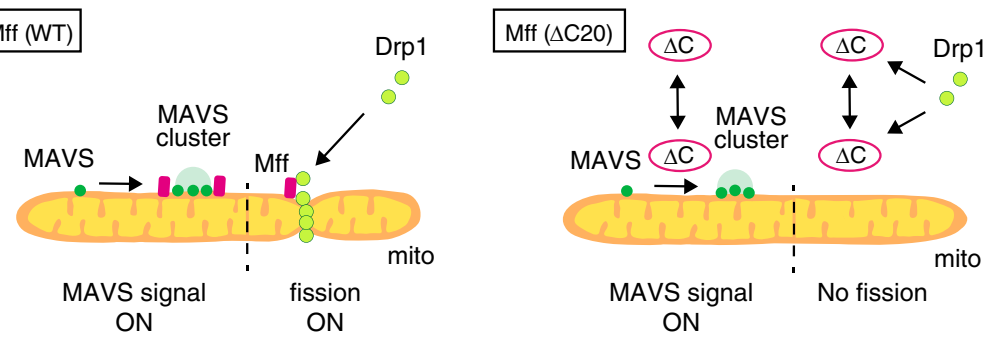

$\mathrm{Mff}(\Delta \mathrm{N} 100)$
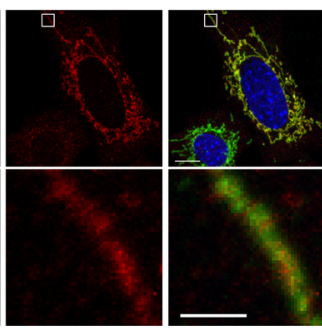

Mff KO MEF

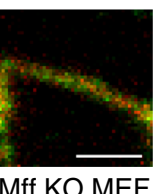

Mff KO MEF

ON

(Fig. 5a, b). We found that the phosphorylation-mimic mutant S146D migrated slower than the phosphorylation-negative mutant S146A in sodium dodecyl sulfate (SDS)-PAGE, similar to phosphorylated and non-phosphorylated Mff (WT), respectively (Fig. 5b). Under the infectious condition, S146A rescued cytokine induction in Mff KO MEFs (Fig. 5c, d), without inducing apparent mitochondrial fission (Fig. 5e), but it induced MAVS cluster formation (images in Fig. 5f, quantification in Fig. 5g). In contrast, S146D rather inhibited cytokine induction (Fig. 5c, d), which induced mitochondrial fission (Fig. 5e), without inducing MAVS cluster formation (Fig. 5f, g). Unexpectedly, stably expressed Mff (WT) failed to activate the cytokine induction (Supplementary Fig. 4e, f), different from those of transiently expressed Mff (WT) that rescued antiviral response in Mff KO 
Fig. 3 Mff regulates MAVS-mediated antiviral response independent of mitochondrial fission. a Scheme of murine Mff deletion constructs. The amino acid numbering scheme used in the constructs corresponds to splice isoform 8 of Mff ${ }^{13}$. TM: transmembrane domain. $\mathbf{b}$ Confocal microscopic images of Mff and mitochondria. Mff KO MEFs were transfected with expression vectors for FLAG-tagged Mff $(\mathrm{WT}), \mathrm{Mff}(\Delta \mathrm{C} 20)$, or Mff $(\Delta \mathrm{N} 100)$, stained with MitoTracker Red (mitochondria; red), and immunostained with an anti-FLAG antibody (green). Nuclei were stained with DAPI (blue). Scale bars: $10 \mu \mathrm{m}$. c qRT-PCR analysis of IFN- $\beta$ mRNA in WT MEFs, Mff KO MEFs, and Mff KO MEFs transfected with an empty vector or expression vectors for FLAG-tagged Mff (WT), Mff ( $\Delta$ C20), or Mff ( $\Delta$ N100). Cells were transfected with or without poly $(\mathrm{l}: \mathrm{C})-\mathrm{LMW}(1 \mu \mathrm{g} / \mathrm{mL})$ for $8 \mathrm{~h}$. Data represent means $\pm \mathrm{SEM}$ from three independent experiments (vector, WT, $\Delta \mathrm{C} 20, \Delta \mathrm{N} 100: n=6,4,4,5, P$ value; two-tailed unpaired $t$ test). $\mathbf{d}$ Immunoblot analyses of endogenous and exogenous Mff and Mff phosphorylation in the cells listed in c. The indicated letters (a, b, c) correspond to the endogenous Mff bands observed in WT MEFs. e Confocal microscopic images of MAVS and Mff. Mff KO MEFs transfected with expression vectors for Mff (WT), Mff ( $\Delta$ C20), or Mff ( $\Delta$ N100) were immunostained with anti-MAVS (green) and anti-Mff (red) antibodies. Nuclei were stained with Hoechst 33342 (blue). A magnified view of the boxed region is shown below. Scale bars: $10 \mu \mathrm{m}$ (whole) and $2 \mu \mathrm{m}$ (magnified). f Magnified images of MAVS on mitochondria in Mff KO MEFs cotransfected with each Mff mutant and a mitochondrial marker mitoRFP (red). Cells were immunostained with anti-MAVS antibody (green). Scale bars: $2 \mu \mathrm{m} . \mathbf{g}$ Co-localization ratio between MAVS and mitoRFP in WT MEFs or Mff KO MEFs co-transfected with mitoRFP and an empty vector or each Mff mutant. Data represent means \pm SEM (WT-vector, Mff KO-vector, Mff KO-WT, Mff KO- $\Delta$ C20, Mff KO- $\Delta$ N100: $n=15,13,16,16,16, P$ value; two-tailed unpaired $t$ test). $\mathbf{h}$ Working hypothesis for the coupling of the distribution of MAVS and mitochondrial fission by Mff. mito: mitochondria. Immunoblot and microscopic analyses were repeated independently three times with similar results. Source data are provided as a Source data file.

a

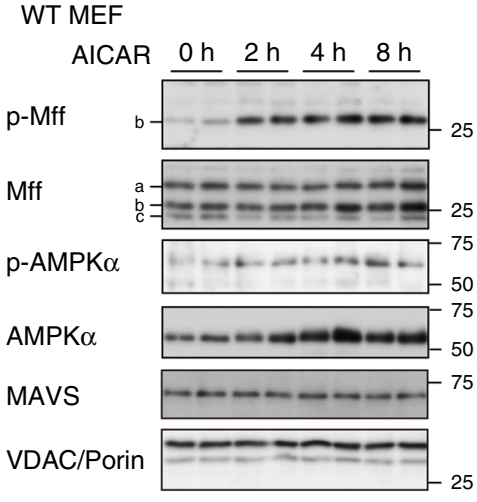

$(\mathrm{kDa})$

d

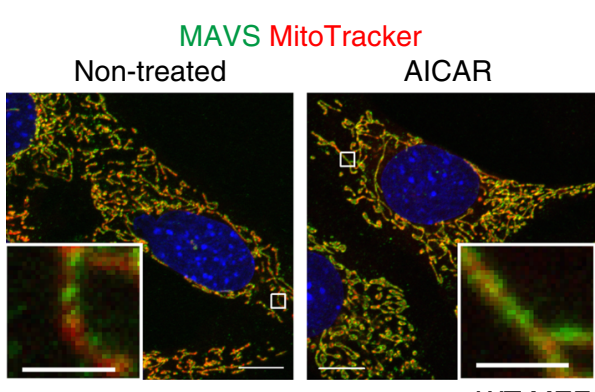

WT MEF b

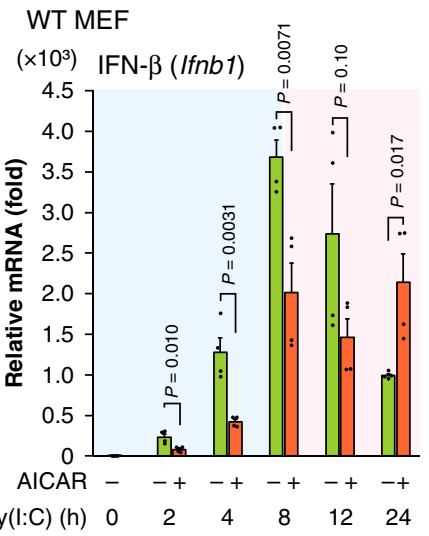

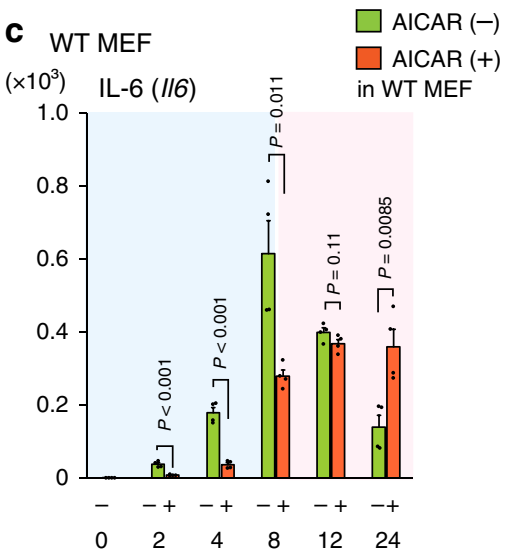

f

Mff KO MEF

$\left(\times 10^{3}\right) \quad$ IFN- $\beta($ Ifnb1)

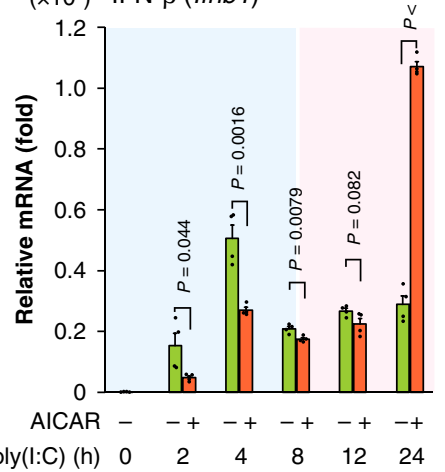

\section{Mff KO MEF}

$\left(\times 10^{3}\right) \quad \mathrm{IL}-6(I / 6)$

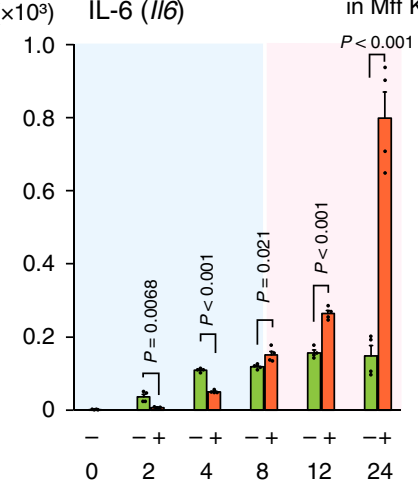

Fig. 4 The acute antiviral response is decreased in AICAR-treated cells with Mff phosphorylation. a WT MEFs were treated with the AMPK activator $\operatorname{AICAR}(2 \mathrm{mM})$ for the indicated times. Whole-cell lysates were analyzed by immunoblotting using antibodies against the indicated proteins. The indicated letters $(a, b, c)$ correspond to the endogenous Mff bands observed in WT MEFs. b, c qRT-PCR analyses of IFN- $\beta$ (b) and IL-6 (c) mRNA in WT MEFs transfected with poly $(\mathrm{I}: \mathrm{C})$-LMW $(1 \mu \mathrm{g} / \mathrm{mL})$ in the absence $(-)$ or presence $(+)$ of AICAR $(2 \mathrm{mM}$, pretreated for $2 \mathrm{~h})$ for the indicated times. $\mathbf{d}$ WT MEFs were stained with MitoTracker Deep Red (mitochondria; red) and then treated with or without AICAR ( $2 \mathrm{mM})$ for $8 \mathrm{~h}$. After fixation and permeabilization, cells were immunostained with an anti-MAVS antibody (green). Nuclei were stained with Hoechst 33342 (blue). Scale bars: $10 \mu \mathrm{m}$ (whole) and $2 \mu \mathrm{m}$ (inset). e, $\mathbf{f}$ qRT-PCR analyses of IFN- $\beta$ (e) and IL-6 (f) mRNA in Mff KO MEFs transfected with poly(I:C)-LMW (1 $\mu \mathrm{g} / \mathrm{mL})$ in the absence (-) or presence $(+)$ of AICAR ( $2 \mathrm{mM}$, pretreated for $2 \mathrm{~h})$ for the indicated times. The blue shaded parts of the graphs $(0-8 \mathrm{~h})$ represent the acute phase after poly $(\mathrm{l}: \mathrm{C})$, and the red shaded parts $(8-24 \mathrm{~h})$ represent the tolerant phase in $\mathbf{b}, \mathbf{c}, \mathbf{e}, \mathbf{f}$. Data represent means \pm SEM from two independent experiments $(n=4, P$ values; two-tailed unpaired $t$ test) in $\mathbf{b}, \mathbf{c}, \mathbf{e}, \mathbf{f}$. Immunoblot analyses and all microscopic analyses were repeated independently more than three times with similar results. Source data are provided as a Source data file. 
a

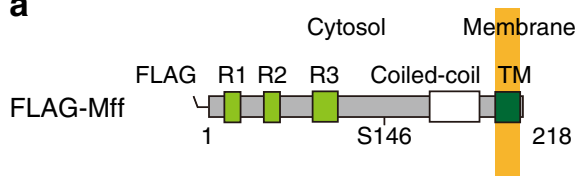

Phospho-mimic: S146D (SD)

Phospho-negative: S146A (SA)

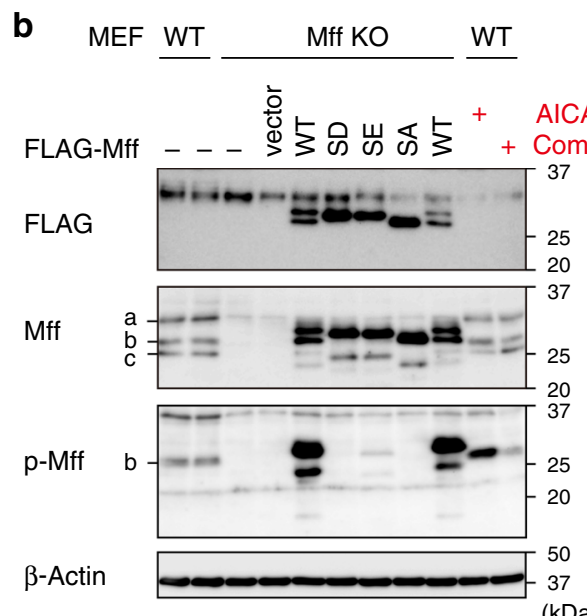

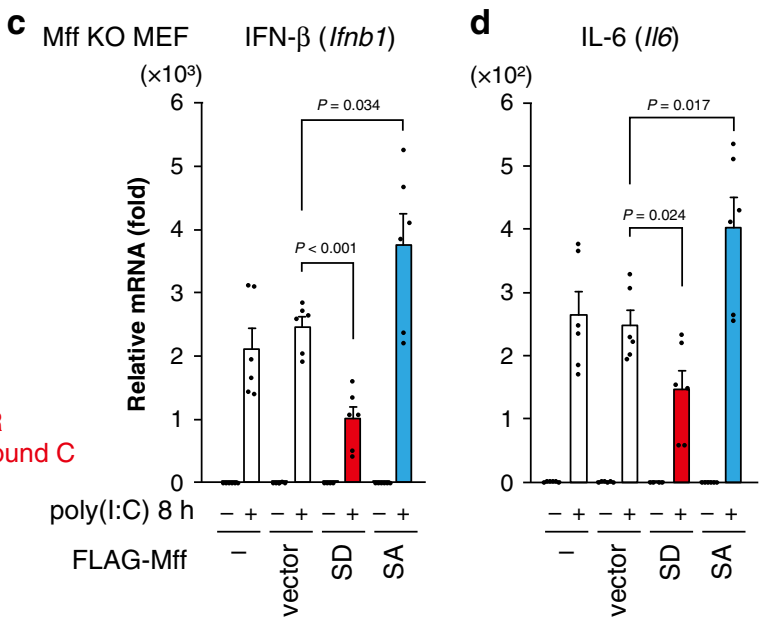
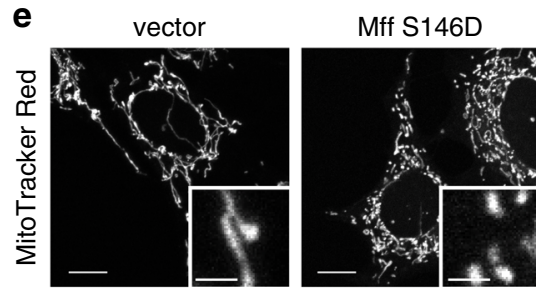

Mff S146A

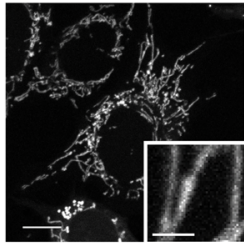

Mff KO MEF
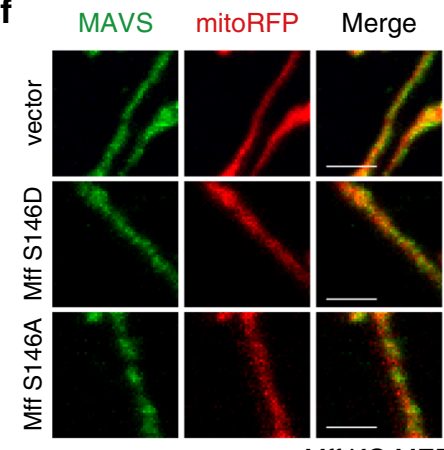

g

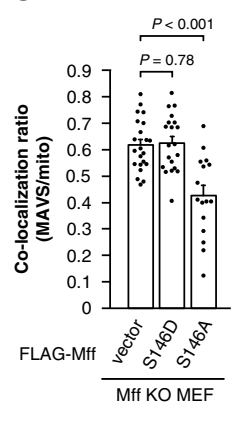

h

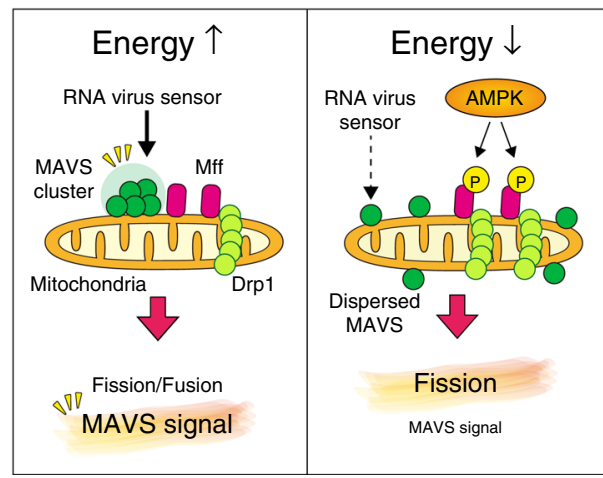

Fig. 5 Mff phosphorylation impairs mitochondrial antiviral response. a Scheme of the murine phosphorylation-negative/mimic Mff constructs used in this study. A point mutation was introduced into FLAG-tagged Mff (WT) at S146 as indicated. b Mff KO MEFs stably expressing each Mff construct were analyzed by immunoblotting using anti-FLAG, anti-Mff, and anti-phospho-Mff antibodies. SD: S146D, SE: S146E, SA: S146A. WT MEFs treated with AICAR (AMPK activator: $2 \mathrm{mM}$ ) or compound C (AMPK inhibitor: $20 \mu \mathrm{M}$ ) for $2 \mathrm{~h}$ were used as controls. c, d qRT-PCR of IFN- $\beta$ (c) and IL-6 (d) mRNA in Mff KO MEFs stably expressing each Mff construct with or without poly $(\mathrm{I}: \mathrm{C})$-LMW $(1 \mu \mathrm{g} / \mathrm{mL})$ transfection for $8 \mathrm{~h}$. Data represent means \pm SEM of three independent experiments ( $n=6, P$ values; two-tailed unpaired $t$ test). e Representative live-cell images of MitoTracker Red (mitochondria, white) in Mff KO MEFs stably expressing each Mff construct. Scale bars: $10 \mu \mathrm{m}$ (whole) and $2 \mu \mathrm{m}$ (inset). f Magnified images of MAVS on mitochondria in Mff KO MEFs stably expressing each Mff construct. The cells were transiently transfected with a mitochondrial marker mitoRFP (red) and immunostained with an antiMAVS antibody (green). Scale bars: $2 \mu \mathrm{m}$. $\mathbf{g}$ Co-localization ratio between MAVS and mitoRFP in Mff KO MEFs stably expressing each Mff construct. Data represent means \pm SEM (vector, SD, SA: $n=22,20,16, P$ values; two-tailed unpaired $t$ test). $\mathbf{h}$ Working hypothesis for the energetic regulation of the mitochondrial antiviral response by Mff phosphorylation. Immunoblot analyses and all microscopic analyses were repeated independently more than three times with similar results. Source data are provided as a Source data file.

cells (Fig. 3c). Stably expressed Mff (WT) was excessively phosphorylated at S146 (Fig. 5b), which should cause impaired antiviral response, although the molecular details remain unknown. Thus Mff phosphorylation by AMPK repressed the antiviral response, concomitant with the activation of mitochondrial fission (model: Fig. 5h).

Mitochondrial metabolism controls MAVS via Mff phosphorylation. MAVS signaling is repressed in various conditions affecting mitochondria such as the depletion of mitochondrial fusion factors ${ }^{30}$, treatment with respiratory inhibitors ${ }^{30,31}$, or in cells with mutated mtDNA ${ }^{31}$. In addition, RNAi of mitochondrial fusion proteins ${ }^{49}$, but not mitochondrial fission proteins ${ }^{50}$, causes respiratory dysfunction in various types of cultured cells. However, the molecular mechanism by which mitochondrial dynamics and activity modulate the antiviral response is less well understood. Here we examined the role of the AMPK-Mff axis in the induction of the antiviral response under various mitochondrial respiration-stressed conditions. 
a

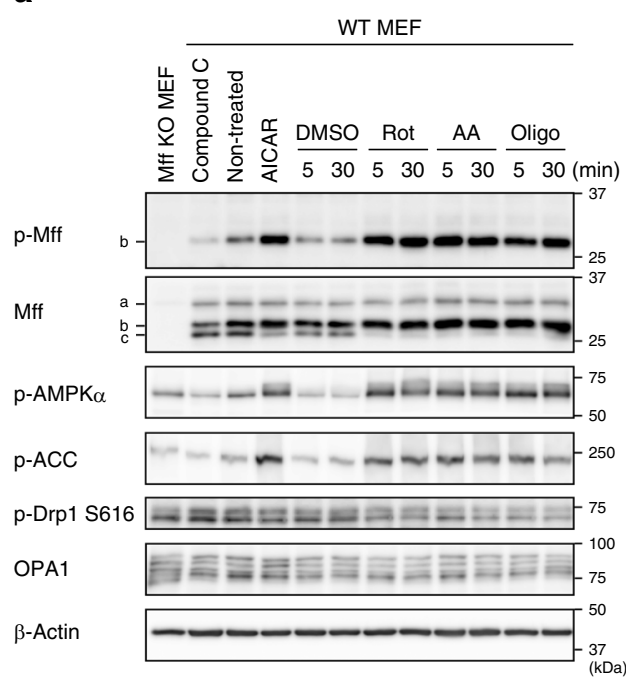

d

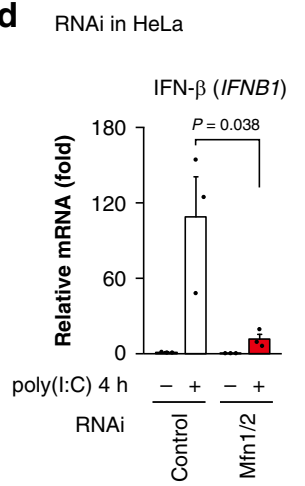

b

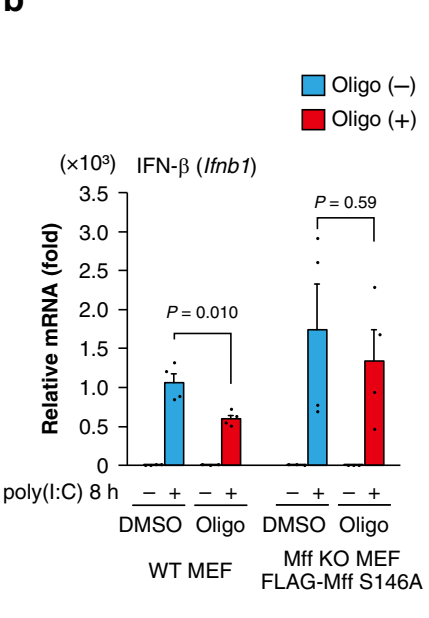

c

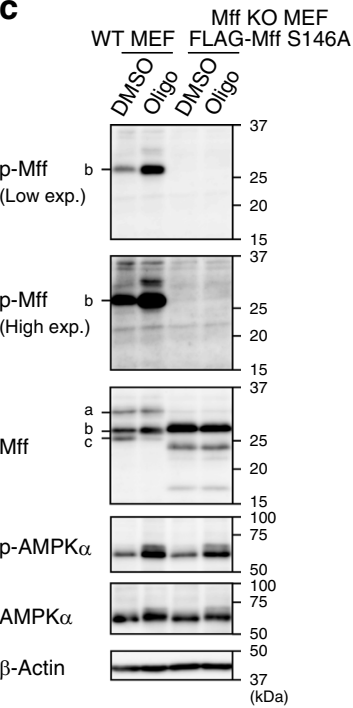

f
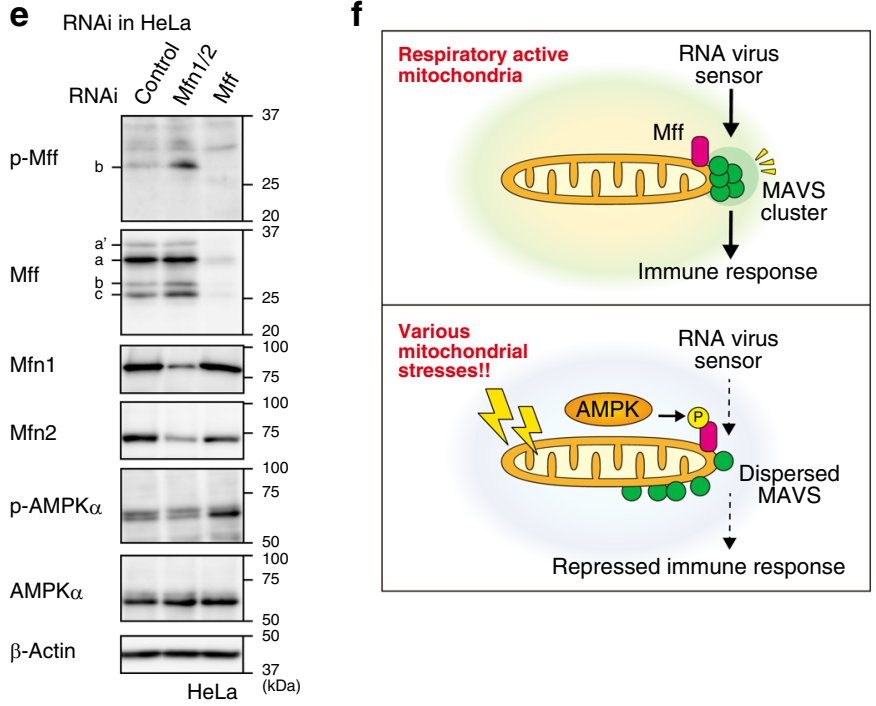

Fig. 6 Mitochondrial dysfunction leads to Mff phosphorylation and impaired antiviral response. a WT MEFs were treated with the respiratory inhibitors rotenone (Rot, $0.5 \mu \mathrm{M})$ or antimycin $\mathrm{A}(\mathrm{AA}, 1 \mu \mathrm{M})$ or the ATP synthase inhibitor oligomycin (Oligo, $1 \mu \mathrm{M})$ for 5 or 30 min. Whole-cell lysates were analyzed by immunoblotting using antibodies against the indicated proteins. AICAR ( $2 \mathrm{mM})$ or compound $\mathrm{C}(20 \mu \mathrm{M})$ was used as an AMPK activator or inhibitor, respectively. DMSO: dimethyl sulfoxide. $\mathbf{b}$ qRT-PCR analysis of IFN- $\beta$ mRNA with or without poly(I:C)-LMW in WT MEFs and Mff KO MEFs stably expressing FLAG-Mff S146A. Cells were pretreated with or without oligomycin (Oligo, $1 \mu \mathrm{M}$ ) for 30 min and then transfected with poly(I:C)-LMW (1 $\mu \mathrm{g} / \mathrm{mL}$ ) in the absence $(-)$ or presence $(+)$ of oligomycin for $8 \mathrm{~h}$. Data represent means \pm SEM of two independent experiments $(n=4, P$ values; twotailed unpaired $t$ test). $\mathbf{c}$ Immunoblot analyses of Mff and Mff phosphorylation in WT MEFs and Mff KO MEFs stably expressing FLAG-Mff S146A with or without oligomycin $(1 \mu \mathrm{M}, 30 \mathrm{~min})$. The indicated letters $(\mathrm{a}, \mathrm{b}, \mathrm{c})$ correspond to the endogenous Mff bands observed in WT MEFs. d HeLa cells were transfected with control or Mfn1/2 siRNA and cultured for $72 \mathrm{~h}$. The cells were transfected with or without poly $(\mathrm{I}: \mathrm{C})-\mathrm{LMW}(1 \mu \mathrm{gg} / \mathrm{mL})$ for $4 \mathrm{~h}$, and IFN- $\beta$ induction was analyzed by qRT-PCR. Data represent means \pm SEM of three independent experiments $(n=3, P$ values; two-tailed unpaired $t$ test).

e Analysis of Mff phosphorylation by immunoblotting using anti-phospho-Mff and anti-Mff antibodies in HeLa cells with the indicated RNAi. The efficiency of RNAi and AMPK activation were also examined by each antibody. $\mathbf{f}$ Working hypothesis for how mitochondrial dysfunction leads to the suppression of the antiviral response by the AMPK-Mff axis. Immunoblot analyses were repeated independently three times with similar results. Source data are provided as a Source data file.

Treatment with respiratory inhibitors induced the phosphorylation of Mff by AMPK (Fig. 6a) as well as acetyl-CoA carboxylase (ACC) phosphorylation, as reported previously ${ }^{38}$. As expected, treatment with oligomycin, an inhibitor of mitochondrial ATP synthase, induced Mff phosphorylation (Fig. 6a) and repressed the induction of IFN- $\beta$ (Fig. 6b), suggesting that the phosphorylation of Mff by AMPK is activated by reduced ATP production under mitochondrial dysfunction, thereby impairing the antiviral response. We also confirmed that the protein band shift (from "c" to "b" in Fig. 6a) induced by AICAR and respiratory inhibitors corresponded with Mff phosphorylation. In Mff KO MEFs expressing the phosphorylation-negative mutant S146A, oligomycin treatment had a reduced impact on the antiviral response (Fig. 6b), even when AMPK was activated (Fig. 6c), confirming that the AMPK-dependent phosphorylation of Mff repressed the antiviral response under mitochondrial dysfunction (Fig. 6f). 
Next, we examined the role of mitochondrial dynamics by fusion and fission in the antiviral response. As shown above, Drp1 RNAi caused mitochondrial elongation (Supplementary Fig. 4j) but had no effect on the antiviral response (Fig. 11), consistent with the notion that it only weakly affects respiratory activity $^{50}$. We further found that the antiviral response in Drp1depleted cells was significantly repressed by AICAR treatment as in control cells (Supplementary Fig. 4a-d), while mitochondria keep highly elongated structures (Supplementary Fig. 4a), suggesting again that AMPK-Mff should have important role in the antiviral response independent of mitochondrial fission. It is noteworthy that the phosphorylation levels of AMPKa and the AMPK substrate ACC in WT MEFs were comparable with those of Mff KO MEFs (Fig. 6a), suggesting that Mff repression should not have a drastic impact on mitochondrial energetics. In contrast, double RNAi of Mfn1 and Mfn2, which has been reported to affect respiration ${ }^{49,51}$, led to an efficient Mff phosphorylation (Fig. 6e) and a defective antiviral response (Fig. 6d) as reported ${ }^{30}$. Under Mfn1/2 depletion, the p-AMPKa band migrated more slowly, possibly due to phosphorylation, compared with control or Mff-depleted cells (Fig. 6e), also confirming that MAVS signaling should be regulated under AMPK. These data suggested that the repressed antiviral signaling in Mfn1/Mfn2-deficient cells should be caused by the AMPKdependent Mff phosphorylation. We further investigated how mitochondria affect Mff phosphorylation by AMPK. In contrast to stably expressed Mff (Fig. 5b), transiently expressed Mff (WT) was only partly phosphorylated at S146 (Fig. 3d) and activated the antiviral response (Fig. $3 \mathrm{c})$. In the same condition, Mff $(\Delta \mathrm{N} 100)$, which localized to mitochondria, was efficiently phosphorylated on mitochondria, although Mff $(\Delta \mathrm{C} 20)$, which localized to the cytoplasm, was only rarely phosphorylated (Fig. 3d). These data are well consistent with the efficient antiviral activity of Mff $(\Delta \mathrm{C} 20)$ (Fig. 3c), even when localized in the cytoplasm. From these data, we concluded that the mitochondrial localization of Mff is important for the AMPK-dependent regulation. Taken together, the MAVS-mediated antiviral response is suppressed under various respiration-stressed conditions via Mff phosphorylation by AMPK, possibly sensing the production of ATP by mitochondria (model: Fig. 6f).

Mff inactivates MAVS at the late phase of antiviral response. Our data suggested that the induction and termination of the temporal innate immune response are controlled by Mff (Fig. 1d-f). To investigate the mechanism underlying the repression of the MAVS-mediated innate immune response at the tolerant phase, we examined time course of the MAVS distribution during the antiviral response (Fig. 7a, b). The MAVS clusters observed before the poly(I:C) introduction (Fig. 2a-c) were maintained for 1 and $4 \mathrm{~h}$ after poly(I:C) introduction (Fig. 7a). In the early phase of the antiviral response in WT MEFs, many MAVS clusters were observed on mitochondria (Fig. 7a). In contrast, by further prolonged stimulation (after $\geq 8 \mathrm{~h}$ ), the MAVS clusters were lost from the mitochondrial surface (Fig. 7a, b), although no AMPK activation was observed at the late stage (after $8 \mathrm{~h}$ ) (Supplementary Fig. 2d). At the late stage, many MAVS signals were observed in the cytoplasm (Fig. 7a). After prolonged antiviral signaling, the cytoplasmic MAVS signals were not observed in Mff KO MEFs but were observed in Drp1 KO MEFs (Fig. 7b), suggesting that Mff mediates the disorganization of the mitochondrial MAVS clusters independently of mitochondrial fission. Cell count analysis also confirmed our conclusion that Mff, but not Drp1, regulates the formation of the cytoplasmic MAVS (Fig. 7c). Collectively, Mff plays important roles in the distribution of MAVS, which should mediate the acute and temporal antiviral response.

\section{Discussion}

Mitochondria are derived from the endosymbiosis of bacteria; however, they also act as a platform for innate immune signaling through MAVS ${ }^{21-24}$. This antiviral platform is not static because MAVS changes its oligomeric status ${ }^{25}$, and mitochondrial membranes are considered to be dynamic as they undergo frequent cycles of fusion and fission ${ }^{5}$. To investigate the dynamic features of the mitochondrial innate immune signaling pathway, we focused on Mff, which is located on the mitochondrial OM and regulates mitochondrial membrane dynamics under cellular signaling ${ }^{13,38}$. Here we found a role for Mff that was independent of mitochondrial fission and mediates the anti-RNA virus response with MAVS.

RNA virus infection induces mitochondrial morphological changes of elongation ${ }^{27}$ or fission ${ }^{44}$. Mfn2 directly interacts with and inhibits MAVS ${ }^{26}$; however, it is unknown how mitochondrial fusion and fission affect the MAVS-mediated innate immune response. From careful morphological and biochemical analyses, we found that mitochondrial morphology changed over time after the introduction of RNA, from fragmentation to elongation. However, we also showed that mitochondrial fission does not directly affect the antiviral response because antiviral cytokines were induced under mitochondrial fission-deficient conditions (Figs. 11, 3, and 5); instead, Mff plays a critical role in the mitochondrial distribution of MAVS (Fig. 2). Mff and MAVS are both anchored to the $\mathrm{OM}$ via their C-terminal membrane anchor domains. The cytoplasmic domain of Mff recruits Drp1 to mitochondrial fission sites via a transient interaction, which mediates the formation of Drp1 oligomers to constrict and divide the mitochondrial membrane ${ }^{5}$. In this analogy, we speculated that Mff should transiently associate with MAVS on the OM and affect its conformation and/or oligomeric status to form activated MAVS clusters, as we showed in CN-PAGE analysis that Mff regulates the oligomeric status of MAVS (Supplementary Fig. 2c). From biochemical analysis, MAVS forms prion-like oligomeric aggregates that are active on mitochondria ${ }^{25,52}$; even the size of MAVS oligomers is not large in vivo ${ }^{43}$ compared with an estimation from an in vitro study ${ }^{25}$. Here we found that MAVS forms dot-like clusters (Fig. 2), as reported previously ${ }^{43}$. These clusters should be a type of membrane subdomain on the mitochondrial surface for the activation of MAVS. Furthermore, under chronic virus infection, Mff mediates the disorganization of mitochondrial MAVS clusters (Fig. 7), which should lead to immune tolerance. In some cases, an acute and drastic immune response efficiently protects cells from virus infection; however, an excessive and prolonged response could damage host cells ${ }^{42}$. A previous report indicated that apoptosis-related caspases cleave MAVS during the antiviral response ${ }^{53}$, but we failed to find degradation products of MAVS (see Fig. 1g); instead, we found changes in the distribution of MAVS at the signal-termination stage (Fig. 7), although Mff phosphorylation was not significantly enhanced (Supplementary Fig. 2d). Further detailed analysis is needed to determine how MAVS forms clusters and detaches from the mitochondrial membrane mediated by Mff. The dynamic movement of MAVS clusters represents a good target for evaluating the immune response in vivo and for manipulating the antiviral response.

Here we found that the antiviral response is repressed under respiratory dysfunction via Mff phosphorylation by AMPK (Figs. 4-6). It has been reported that the MAVS-dependent antiviral response is repressed by various mitochondrial changes, such as mitochondrial fusion deficiency, treatment with an 
a WT MEF

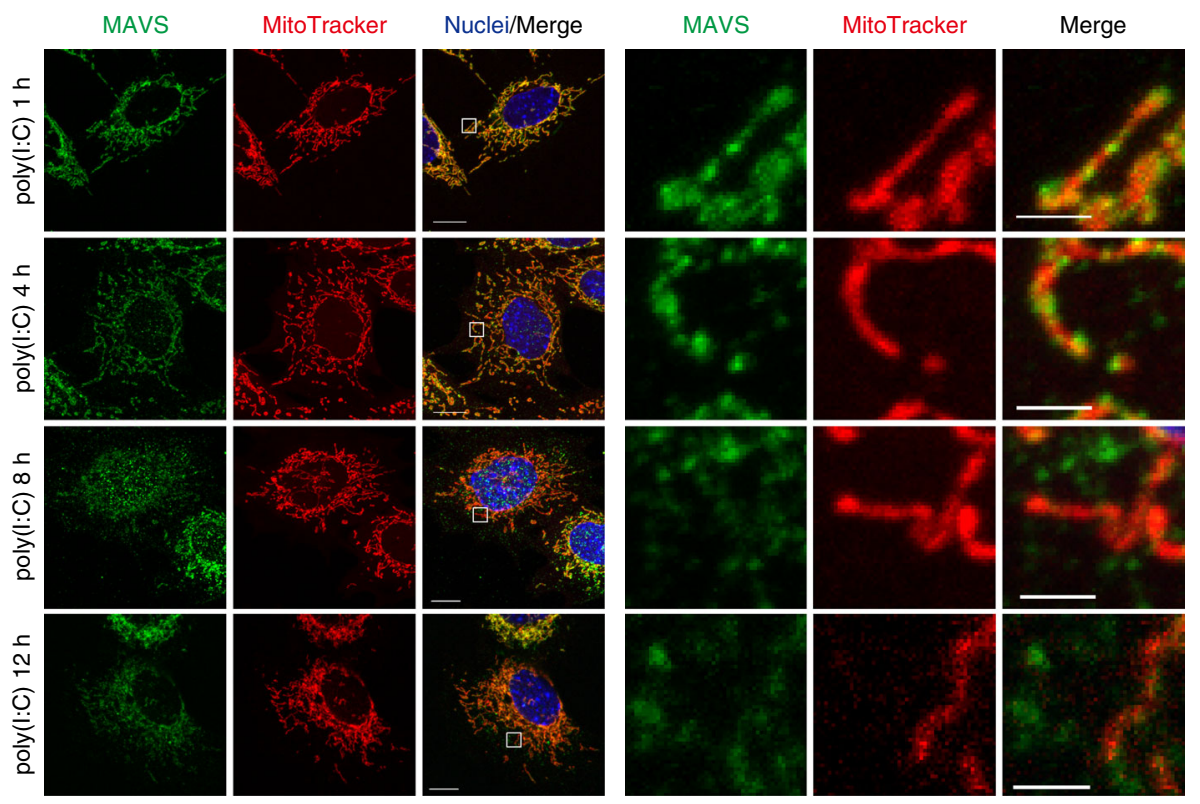

b MAVS/MitoTracker/Nuclei

d
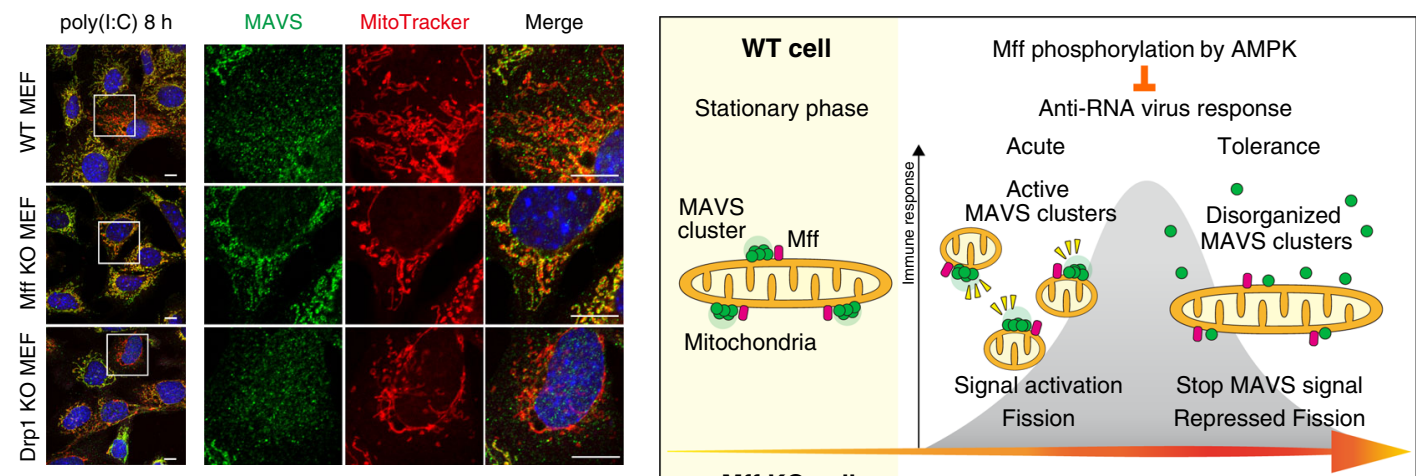

C

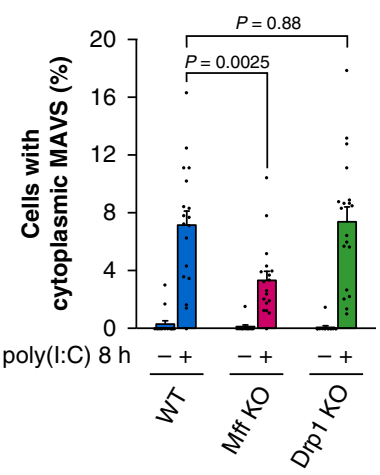

\section{Mff KO cell}

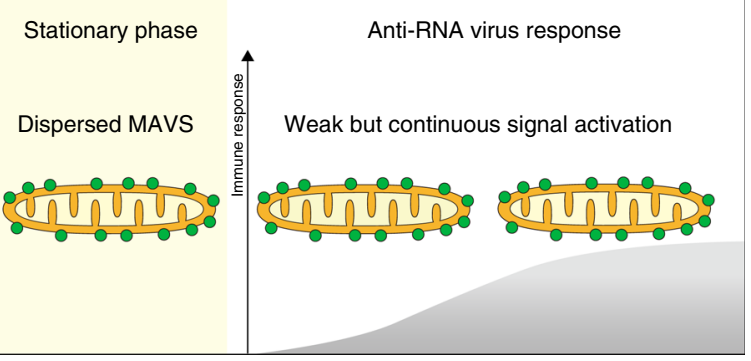

Fig. 7 Clearance of mitochondrial MAVS clusters by Mff. a Confocal microscopic images of MAVS and mitochondria. WT MEFs were transfected with poly (I:C)-LMW $(1 \mu \mathrm{g} / \mathrm{mL})$ for the indicated times. Cells were stained with MitoTracker Red (mitochondria; red) and immunostained with an anti-MAVS antibody (green). Nuclei were stained with Hoechst 33342 (blue). A magnified view of the boxed region is shown in the right panel. Scale bars: $10 \mu \mathrm{m}$ (whole) and $2 \mu \mathrm{m}$ (magnified). b WT, Mff KO, and Drp1 KO MEFs were stained with MitoTracker Red (mitochondria; red) and immunostained with an antiMAVS antibody (green) with poly $(\mathrm{I}: \mathrm{C})-\mathrm{LMW}(1 \mu \mathrm{g} / \mathrm{mL})$ transfection for $8 \mathrm{~h}$. Nuclei were stained with DAPI (blue). A magnified view of the boxed region is shown in the right panel. Scale bars: $10 \mu \mathrm{m}$. c The percentage of cells with cytoplasmic MAVS (MAVS signal without MitoTracker Red) was calculated. Data represent means \pm SEM of three independent experiments (WT, Mff KO, Drp1 KO; $n=18,18,19, P$ values; two-tailed unpaired $t$ test). d Working hypothesis for the mitochondrial membrane subdomains with MAVS clusters in antiviral innate immunity, dynamically regulated by the mitochondrial fission factor Mff and AMPK. All microscopic analyses were repeated independently more than three times with similar results. Source data are provided as a Source data file. 
uncoupler, and mtDNA mutations ${ }^{30,31}$. Under these conditions, mitochondrial respiratory activity should be repressed; thus the reduced supply of ATP from mitochondria enhances AMPK activity around mitochondria, which leads to efficient Mff phosphorylation and repression of the antiviral response. Thus Mff links mitochondrial energy production to the innate immune response on mitochondria. These findings provide a clue to solve why the immune response is activated on mitochondria. Mff is one of the main substrates of AMPK in a part of the energy sensor/regulation system ${ }^{38}$, although the physiological function of this energy-dependent modification is less well understood. It seems reasonable to assume that, under pathogenic as well as energy-limiting conditions, cells manage to survive by preserving energy to suppress drastic cytokine synthesis and by promoting energy production to activate mitochondrial fission. Mitochondria are the location of ATP production; thus it seems appropriate that the ATP-sensing system is located on the mitochondrial surface because a proportion of AMPK is localized on mitochondria ${ }^{54}$. Our findings should provide a missing link beyond metabolism to mitochondrial signaling and immunity and point to the possibility that Mff is a therapeutic target for energylimiting pathology. However, we need further analysis of the molecular mechanism by which Mff regulates the distribution and function of MAVS and how and why Mff regulates antiviral signaling in response to energy status. It was also reported that repression of Mfn2 alone increases the MAVS-mediated antiviral response $^{26}$, possibly by the direct suppression of MAVS by Mfn2, which appears to be a different mechanism to that reported here, indicating that further detailed analysis is required. Our report reveals an important aspect of the relationship between innate immunity and metabolism, in which mitochondrial dynamics is the key regulator of multifunctional mitochondria.

\section{Methods}

Cell culture and transfection. MEFs and HeLa cells were cultured in Dulbecco's modified Eagle's medium (043-30085; Wako, Osaka, Japan) supplemented with $10 \%$ fetal bovine serum. Drp1 KO MEFs ${ }^{6}$ and Mff KO HeLa cells ${ }^{16}$ were generated previously. Mff KO MEFs were generated as described in Supplementary Methods. Lipofectamine LTX (Life Technologies, Carlsbad, CA) and OptiMEM (Gibco) were used for the transfection of poly(I:C)-LMW, poly(I:C)-HMW (\#tlrl-picw, \#tlrl-pic; InvivoGen, San Diego, CA), and poly(dI:dC) (P4929; Sigma-Aldrich). Lipofectamine LTX with PLUS or Lipofectamine 2000 (Life Technologies) was used for the transfection of plasmid DNA. An empty vector was used to maintain equal amounts of DNA among wells (100 ng in Fig. 1m, $1.25 \mu \mathrm{g}$ in Fig. 3). Lipofectamine RNAiMAX (Life Technologies) was used for RNAi and small interfering RNA (siRNA) targeting human Mff (siGENOME siRNA SMARTpool, M-018261-010005), human MAVS (siGENOME siRNA SMARTpool, M-024237-02-0005), and a non-targeting control siRNA \#2 (D-001210-02-05) were purchased from Dharmacon (Lafayette, CO). Other siRNAs are shown in Supplementary Methods. Cells were transfected with $20 \mathrm{nM}$ siRNA and the medium was replaced after $4 \mathrm{~h}$. At $72 \mathrm{~h}$ post-transfection, the cells were stimulated or analyzed. Knockdown efficiency was determined by immunoblot analysis.

Plasmids. Hemagglutinin (HA)-tagged human MAVS pcDNA3.1 was reported previously $^{26}$. FLAG-tagged mouse Mff (WT), Mff $(\Delta \mathrm{C} 20)$, and Mff $(\Delta \mathrm{N} 100)$ pcDNA3.1 were reported previously ${ }^{13}$. Full-length Mff used in this study corresponds to isoform 4 (NM_001310699.1 in NCBI), Mff-209

(ENSMUST00000160972.7 in Ensemble genome), and splice isoform 8 of $\mathrm{Mff}^{13}$. pSu9(1-69)-RFP (mitoRFP) was reported previously ${ }^{55}$.

RNA virus infection. SeV Cantell strain was purchased from ATCC (VR-907; Manassas, VA) ${ }^{56}$. MEFs were infected with SeV [4 HA units/mL] for 10 and $24 \mathrm{~h}$, and the cell-free supernatants were analyzed by ELISA to measure the amount of IFN- $\beta$.

Construction of plasmids and Mff KO MEFs stably expressing Mff constructs. FLAG-tagged Mff constructs ${ }^{13}$ were subcloned into the BamHI and NotI sites of the pMXs-IP vector ${ }^{57}$. For the phosphorylation-mimic or phosphorylationnegative mutant of Mff, S146 (TCC) of Mff was changed to D (GAC) or A (GCC), respectively. We also constructed S146E (TCC to GAG) of Mff mutant for another phosphorylation-mimic mutant. The primers are listed in Supplementary Table 1. Mutagenesis was carried out using a PrimeSTAR Mutagenesis Basal Kit (Takara
Bio, Inc., Shiga, Japan) and confirmed by sequencing. Mff KO MEFs were transfected with empty or each expression pMXs-IP vector $(1.25 \mu \mathrm{g})$ in a 6-well format by Lipofectamine 2000, the medium was replaced at $4 \mathrm{~h}$, and the cells were further cultured for 24-48 h. All stable cell lines were established by subsequent selection in the presence of puromycin $(2 \mu \mathrm{g} / \mathrm{mL})$ for approximately 10 days and nontransfected parental cells were confirmed to be dead.

Chemicals and antibodies. AMPK activator AICAR ( $2 \mathrm{mM}$, ab120358) and AMPK inhibitor compound C $(20 \mu \mathrm{M}$, ab120843) were purchased from Abcam. Respiratory inhibitors rotenone $(0.5 \mu \mathrm{M})$ or antimycin A $(1 \mu \mathrm{M})$ and ATP synthase inhibitor oligomycin $(1 \mu \mathrm{M})$ were purchased from Sigma-Aldrich. MitoTracker Red CMXRos (100 nM, M7512) and MitoTracker Deep Red FM (100 nM, M22426) were purchased from Molecular Probes. Final concentration of dimethyl sulfoxide was set to $0.5 \%$ during the treatment with compound $\mathrm{C}$, rotenone, antimycin $\mathrm{A}$, or oligomycin. The primary and secondary antibodies used for immunoblotting or immunostaining are listed in Supplementary Table 2. Additional information of materials and antibodies are shown in Supplementary Methods.

Quantitative real-time reverse transcriptase-PCR (RT-PCR). Total RNA was isolated from cells using Sepasol RNA I Super G (Nacalai Tesque, Kyoto, Japan), and cDNA synthesis was performed using a QuantiTect Reverse Transcription Kit (Qiagen, Hilden, Germany). cDNAs were quantified with gene-specific primer pairs (Supplementary Table 1) using TB Green Premix Ex Taq II (Tli RNaseH Plus; Takara) and quantitative real-time RT-PCR was conducted using the ABI7500 system or StepOnePlus (Applied Biosystems, Foster City, CA). Gene expression values were normalized to glyceraldehyde-3-phosphate dehydrogenase (Gapdh) gene expression using the delta-delta $\mathrm{Ct}$ method. For relative gene expression (fold), the control sample in the left-most column of each bar graph was standardized at a value of 1 .

Enzyme-linked immunosorbent assay. MEFs were transfected with poly(I:C)LMW $(1 \mu \mathrm{g} / \mathrm{mL})$, poly(I:C)-HMW $(1 \mu \mathrm{g} / \mathrm{mL})$, or poly(dI:dC) $(1 \mu \mathrm{g} / \mathrm{mL})$, or stimulated with LPS $(100 \mathrm{ng} / \mathrm{mL})$ or bovine serum albumin (BSA)-conjugated palmitate $(800 \mu \mathrm{M})$ for $8 \mathrm{~h}$. The culture supernatants were collected and centrifuged to remove nonadherent cells. The concentrations of mouse IFN- $\beta$ (\#42400-1; PBL Assay Science, Piscataway, NJ, or MIFNB0; R\&D Systems, Minneapolis, MN) and mouse IL-6 (M6000B; R\&D Systems) were determined by ELISA according to the manufacturers' instructions. The supernatants of SeV-infected MEFs were also examined.

Immunoblotting. The cells were collected at each indicated time point following stimulation. The cells were washed with ice-cold phosphate-buffered saline (PBS) twice, and lysed on ice in lysis buffer $(20 \mathrm{mM}$ Tris- $\mathrm{HCl} \mathrm{pH} 7.6,150 \mathrm{mM} \mathrm{NaCl}, 2$ mM EDTA, 0.5\% NP-40) with a protease inhibitor cocktail tablet and a phosphatase inhibitor cocktail tablet (Roche Molecular Biochemicals, Mannheim, Germany). The lysates were sonicated and clarified by centrifugation at $16,500 \times g$ at $4{ }^{\circ} \mathrm{C}$ for $10 \mathrm{~min}$. Equal amount protein samples were added to $2 \times$ Laemmli sample buffer (\#161-0737; Bio-Rad, Hercules, CA) with 2-mercaptoethanol (2-ME) or $4 \times$ sample buffer $(0.25 \mathrm{M}$ Tris- $\mathrm{HCl} \mathrm{pH} 6.8,4 \%$ glycerol, $8 \%$ SDS, $0.008 \%$ bromophenol blue) with $20 \% 2-\mathrm{ME}$ and boiled at $98^{\circ} \mathrm{C}$ for $5 \mathrm{~min}$. The samples were separated by SDS-PAGE and transferred to a polyvinylidene difluoride membrane (GE Healthcare or Immobilon-P from Millipore, Burlington, MA). Primary and secondary antibodies were diluted in Can Get Signal Solution 1 and 2 (Toyobo Co., Ltd., Osaka, Japan) or TBST containing 5\% BSA or 5\% non-fat dry milk as shown in Supplementary Table 2. Detection was carried out with ECL (RPN2106, GE Healthcare) or Immobilon Western Chemiluminescent HRP Substrate (Millipore) using LAS4000 mini (GE Healthcare) or VersaDoc 5000 (Bio-Rad). Images were analyzed using ImageJ (National Institutes of Health, Bethesda, MD) or ImageQuant (GE Healthcare). Image brightness and contrast were adjusted by ImageQuant or Photoshop CC (Adobe).

Immunostaining and confocal microscopic analysis. The cells were seeded into four-well chamber slides or on coverslips. To visualize mitochondrial network, cells were transiently expressed with mitoRFP $(250 \mathrm{ng}$ ) or stained with MitoTracker Red CMXRos or MitoTracker Deep Red for $15-30 \mathrm{~min}$ at $37^{\circ} \mathrm{C}$ under conditions of $5 \%$ $\mathrm{CO}_{2}$. The cells were fixed in $4 \%$ paraformaldehyde for $15 \mathrm{~min}$ at room temperature and permeabilized with $0.1 \%$ Triton X-100 for $5 \mathrm{~min}$ at room temperature. After blocking with PBS containing 5\% non-fat dry milk for $1 \mathrm{~h}$, the cells were stained with primary antibodies for $1 \mathrm{~h}$ at room temperature or overnight at $4{ }^{\circ} \mathrm{C}$. After washing with PBS, the cells were incubated with Alexa Fluor-labeled secondary antibodies (1:500) for $1 \mathrm{~h}$ at room temperature. Primary and Alexa Fluor-labeled secondary antibodies were diluted in PBS containing 5\% non-fat dry milk or Can Get Signal immunostain (Toyobo) as listed in Supplementary Table 2. After incubation with DAPI for $5 \mathrm{~min}$ or Hoechst 33342 for $10 \mathrm{~min}$, the cells were washed with PBS and mounted with Fluoromount (Diagnostic BioSystems, Inc., Pleasanton, CA) or SlowFade Gold (Invitrogen). Images $(1024 \times 1024$ pixels) were acquired using a LSM700 (Zeiss, Oberkochen, Germany) using $\times 63 / 1.4$ oilimmersion lens. For Fig. $7 c$, images were acquired with $\times 20$ objective. The image data were analyzed using ZEN 2010 (Zeiss). Z-stacked images $(z=0.25-0.37 \mu \mathrm{m}$, 
5-9 images) were projected in Figs. 2a-c, 3e, f, 4d, 5f, and 7a and Supplementary Figs. $1 \mathrm{c}-\mathrm{d}, \mathrm{f}, 3$, and $4 \mathrm{~h}$.

For live-cell imaging, the cells were seeded in a collagen type I-coated $35-\mathrm{mm}$ glass-based dish (4970-011, Iwaki; Asahi Glass Co., Ltd., Tokyo, Japan) and stained for $15 \mathrm{~min}$ at $37^{\circ} \mathrm{C}$ with MitoTracker Red CMXRos and Hoechst 33342 as needed under conditions of $5 \% \mathrm{CO}_{2}$. After washing with culture medium three times, the cells were rested for $30 \mathrm{~min}$ in culture medium containing $50 \mathrm{mM}$ HEPES under $37^{\circ} \mathrm{C}$ and then transfected with poly(I:C)-LMW as needed. Live-cell images $(1024 \times 1024$ pixels $)$ were acquired using a LSM700 using $\times 63 / 1.4$ oil-immersion lens under $37^{\circ} \mathrm{C}$, and the images were processed using ZEN 2010. $Z$-stacked images ( $z=0.37 \mu \mathrm{m}, 3-4$ images) were projected in Fig. 5e and Supplementary Figs. $1 \mathrm{e}$ and $4 \mathrm{a}, \mathrm{g}, \mathrm{j}$.

For quantification of MAVS distribution in fluorescent images, we measured co-localization efficiency between mitochondria and MAVS. The double-staining images $(512 \times 512$ pixels $)$ of mitochondria and MAVS were acquired using a LSM700 and ZEN 2010 program (Zeiss) with a $\times 63 / 1.4$ oil-immersion lens. The threshold of MAVS signal was set as half of the highest intensity in the image, and threshold of mitochondrial marker was set using Otsu' auto threshold algorism in Fiji/ImageJ program. Using these double-staining binary images of MAVS and mitochondrial marker, the ratio of MAVS signal overlapped on mitochondria was measured by co-localization application in ZEN 2010. In Fig. 2e, co-localization ratio between MAVS and Mff or Drp1 in WT MEFs was calculated using each of binary threshold image by Otsu' auto threshold algorism. For analysis of cytoplasmic MAVS, the percentage of cells with cytoplasmic MAVS (MAVS signal without MitoTracker Red) was counted ( $>800$ cells per group).

Statistics and reproducibility. Data are presented as means \pm standard error of the mean of three independent experiments unless otherwise noted. Each $n$ value basically shows biological replicates and repeated measurement was considered to be technical replicates. In quantification of fluorescent images, region of interest was counted as $n$ value. Images are representative results from basically three independent experiments with similar results. Results were analyzed statistically using a two-tailed unpaired Student's $t$ test or Mann-Whitney's $U$ test $(0 \mathrm{~h}$ in Fig. 1i, j). A $P$ value $<0.05$ was considered statistically significant. Statistical analysis was carried out using Statcel4 (OMS). No statistical method was used to predetermine sample size. Samples were blindly and randomly divided to experimental groups. The investigators were not blinded to allocation during the experiments and outcome assessment.

Reporting summary. Further information on research design is available in the Nature Research Reporting Summary linked to this article.

\section{Data availability}

Data supporting the findings of this study are available in the article and its Supplementary files or from the corresponding author upon reasonable request. Source data are provided with this paper.

Received: 16 March 2020; Accepted: 7 October 2020; Published online: 11 November 2020

\section{References}

1. West, A. P., Shadel, G. S. \& Ghosh, S. Mitochondria in innate immune responses. Nat. Rev. Immunol. 11, 389-402 (2011).

2. Nunnari, J. \& Suomalainen, A. Mitochondria: in sickness and in health. Cell 148, 1145-1159 (2012).

3. Chandel, N. S. Evolution of mitochondria as signaling organelles. Cell Metab. 22, 204-206 (2015).

4. Okamoto, K. \& Shaw, J. M. Mitochondrial morphology and dynamics in yeast and multicellular eukaryotes. Annu. Rev. Genet. 39, 503-536 (2005).

5. Chan, D. C. Fusion and fission: interlinked processes critical for mitochondrial health. Annu. Rev. Genet. 46, 265-287 (2012).

6. Ishihara, N. et al. Mitochondrial fission factor Drp1 is essential for embryonic development and synapse formation in mice. Nat. Cell Biol. 11, 958-966 (2009).

7. Wakabayashi, J. et al. The dynamin-related GTPase Drp1 is required for embryonic and brain development in mice. J. Cell Biol. 186, 805-816 (2009).

8. Waterham, H. R. et al. A lethal defect of mitochondrial and peroxisomal fission. N. Engl. J. Med. 356, 1736-1741 (2007).

9. Wang, L. et al. Disruption of mitochondrial fission in the liver protects mice from diet-induced obesity and metabolic deterioration. Diabetologia $\mathbf{5 8}$, 2371-2380 (2015).

10. Yamada, T. et al. Mitochondrial stasis reveals p62-mediated ubiquitination in Parkin-independent mitophagy and mitigates nonalcoholic fatty liver disease. Cell Metab. 28, 588.e5-604.e5 (2018).
11. Wang, Y. et al. Mitochondrial fission promotes the continued clearance of apoptotic cells by macrophages. Cell 171, 331.e22-345.e22 (2017).

12. Gandre-Babbe, S. \& van der Bliek, A. M. The novel tail-anchored membrane protein Mff controls mitochondrial and peroxisomal fission in mammalian cells. Mol. Biol. Cell 19, 2402-2412 (2008).

13. Otera, H. et al. Mff is an essential factor for mitochondrial recruitment of Drp1 during mitochondrial fission in mammalian cells. J. Cell Biol. 191, 1141-1158 (2010)

14. Palmer, C. S. et al. MiD49 and MiD51, new components of the mitochondrial fission machinery. EMBO Rep. 12, 565-573 (2011).

15. Zhao, J. et al. Human MIEF1 recruits Drp1 to mitochondrial outer membranes and promotes mitochondrial fusion rather than fission. EMBO J. 30, 2762-2778 (2011).

16. Otera, H., Miyata, N., Kuge, O. \& Mihara, K. Drp1-dependent mitochondrial fission via MiD49/51 is essential for apoptotic cristae remodeling. J. Cell Biol. 212, 531-544 (2016).

17. Shamseldin, H. E. et al. Genomic analysis of mitochondrial diseases in a consanguineous population reveals novel candidate disease genes. J. Med. Genet. 49, 234-241 (2012).

18. Koch, J. et al. Disturbed mitochondrial and peroxisomal dynamics due to loss of MFF causes Leigh-like encephalopathy, optic atrophy and peripheral neuropathy. J. Med. Genet. 53, 270-278 (2016).

19. Nasca, A. et al. Clinical and biochemical features in a patient with mitochondrial fission factor gene alteration. Front. Genet. 9, 625 (2018).

20. Chen, $H$. et al. Titration of mitochondrial fusion rescues Mff-deficient cardiomyopathy. J. Cell Biol. 211, 795-805 (2015).

21. Kawai, T. et al. IPS-1, an adaptor triggering RIG-I- and Mda5-mediated type I interferon induction. Nat. Immunol. 6, 981-988 (2005).

22. Meylan, E. et al. Cardif is an adaptor protein in the RIG-I antiviral pathway and is targeted by hepatitis C virus. Nature 437, 1167-1172 (2005).

23. Seth, R. B., Sun, L., Ea, C. K. \& Chen, Z. J. Identification and characterization of MAVS, a mitochondrial antiviral signaling protein that activates NF- $\mathrm{BB}$ and IRF3. Cell 122, 669-682 (2005).

24. $\mathrm{Xu}, \mathrm{L}$. G. et al. VISA is an adapter protein required for virus-triggered IFN- $\beta$ signaling. Mol. Cell 19, 727-740 (2005)

25. Hou, F. et al. MAVS forms functional prion-like aggregates to activate and propagate antiviral innate immune response. Cell 146, 448-461 (2011).

26. Yasukawa, K. et al. Mitofusin 2 inhibits mitochondrial antiviral signaling. Sci. Signal. 2, ra47 (2009).

27. Castanier, C., Garcin, D., Vazquez, A. \& Arnoult, D. Mitochondrial dynamics regulate the RIG-I-like receptor antiviral pathway. EMBO Rep. 11, 133-138 (2010).

28. Onoguchi, K. et al. Virus-infection or 5'ppp-RNA activates antiviral signal through redistribution of IPS-1 mediated by MFN1. PLoS Pathog. 6, e1001012 (2010).

29. Chatel-Chaix, L. et al. Dengue virus perturbs mitochondrial morphodynamics to dampen innate immune responses. Cell Host Microbe 20, 342-356 (2016)

30. Koshiba, T., Yasukawa, K., Yanagi, Y. \& Kawabata, S. Mitochondrial membrane potential is required for MAVS-mediated antiviral signaling. Sci. Signal. 4, ra7 (2011).

31. Yoshizumi, T. et al. RLR-mediated antiviral innate immunity requires oxidative phosphorylation activity. Sci. Rep. 7, 5379 (2017).

32. Otera, H., Ishihara, N. \& Mihara, K. New insights into the function and regulation of mitochondrial fission. Biochim. Biophys. Acta 1833, 1256-1268 (2013).

33. Mishra, P. \& Chan, D. C. Metabolic regulation of mitochondrial dynamics. J. Cell Biol. 212, 379-387 (2016)

34. Ishihara, N., Fujita, Y., Oka, T. \& Mihara, K. Regulation of mitochondrial morphology through proteolytic cleavage of OPA1. EMBO J. 25, 2966-2977 (2006).

35. Gomes, L. C., Benedetto, G., Di \& Scorrano, L. During autophagy mitochondria elongate, are spared from degradation and sustain cell viability. Nat. Cell Biol. 13, 589-598 (2011).

36. Rambold, A. S., Kostelecky, B., Elia, N. \& Lippincott-Schwartz, J. Tubular network formation protects mitochondria from autophagosomal degradation during nutrient starvation. Proc. Natl Acad. Sci. USA 108, 10190-10195 (2011).

37. Ducommun, S. et al. Motif affinity and mass spectrometry proteomic approach for the discovery of cellular AMPK targets: identification of mitochondrial fission factor as a new AMPK substrate. Cell. Signal. 27, 978-988 (2015).

38. Toyama, E. Q. et al. AMP-activated protein kinase mediates mitochondrial fission in response to energy stress. Science 351, 275-281 (2016).

39. Wang, A. et al. Opposing effects of fasting metabolism on tissue tolerance in bacterial and viral inflammation. Cell 166, 1512-1525.e12 (2016).

40. Moriyama, M. \& Ichinohe, T. High ambient temperature dampens adaptive immune responses to influenza A virus infection. Proc. Natl Acad. Sci. USA 116, 3118-3125 (2019). 
41. Zhang, W. et al. Lactate is a natural suppressor of RLR signaling by targeting MAVS. Cell 178, 176-189.e15 (2019).

42. Ivashkiv, L. B. \& Donlin, L. T. Regulation of type i interferon responses. Nat. Rev. Immunol. 14, 36-49 (2014).

43. Hwang, M. S. et al. MAVS polymers smaller than $80 \mathrm{~nm}$ induce mitochondrial membrane remodeling and interferon signaling. FEBS J. 286, 1543-1560 (2019).

44. Kim, S. J. et al. Hepatitis C virus triggers mitochondrial fission and attenuates apoptosis to promote viral persistence. Proc. Natl Acad. Sci. USA 111, 6413-6418 (2014).

45. Pandey, S., Kawai, T. \& Akira, S. Microbial sensing by Toll-like receptors and intracellular nucleic acid sensors. Cold Spring Harb. Perspect. Biol. 7, a016246 (2014).

46. Zevini, A., Olagnier, D. \& Hiscott, J. Crosstalk between cytoplasmic RIG-I and STING sensing pathways. Trends Immunol. 38, 194-205 (2017).

47. Rogero, M. M. \& Calder, P. C. Obesity, inflammation, Toll-like receptor 4 and fatty acids. Nutrients 10, 432 (2018).

48. Yasukawa, K., Kinoshita, D., Yaku, K., Nakagawa, T. \& Koshiba, T. The microRNAs miR-302b and miR-372 regulate mitochondrial metabolism via the SLC25A12 transporter, which controls MAVS-mediated antiviral innate immunity. J. Biol. Chem. 295, 444-457 (2020).

49. Chen, H., Chomyn, A. \& Chan, D. C. Disruption of fusion results in mitochondrial heterogeneity and dysfunction. J. Biol. Chem. 280, 26185-26192 (2005).

50. Ban-Ishihara, R., Ishihara, T., Sasaki, N., Mihara, K. \& Ishihara, N. Dynamics of nucleoid structure regulated by mitochondrial fission contributes to cristae reformation and release of cytochrome c. Proc. Natl Acad. Sci. USA 110, 11863-11868 (2013).

51. Chen, H., McCaffery, J. M. \& Chan, D. C. Mitochondrial fusion protects against neurodegeneration in the cerebellum. Cell 130, 548-562 (2007).

52. $\mathrm{Xu}, \mathrm{H}$. et al. Structural basis for the prion-like MAVS filaments in antiviral innate immunity. eLife 3, e01489 (2014).

53. Rebsamen, M., Meylan, E., Curran, J. \& Tschopp, J. The antiviral adaptor proteins Cardif and Trif are processed and inactivated by caspases. Cell Death Differ. 15, 1804-1811 (2008).

54. Zong, Y. et al. Hierarchical activation of compartmentalized pools of AMPK depends on severity of nutrient or energy stress. Cell Res. 29, 460-473 (2019).

55. Eura, Y., Ishihara, N., Yokota, S. \& Mihara, K. Two mitofusin proteins, mammalian homologues of $\mathrm{FZO}$, with distinct functions are both required for mitochondrial fusion. J. Biochem. 134, 333-344 (2003).

56. Yoshinaka, T. et al. Structural basis of mitochondrial scaffolds by prohibitin complexes: insight into a role of the coiled-coil region. iScience 19, 1065-1078 (2019).

57. Yoshii, S. R., Kishi, C., Ishihara, N. \& Mizushima, N. Parkin mediates proteasome-dependent protein degradation and rupture of the outer mitochondrial membrane. J. Biol. Chem. 286, 19630-19640 (2011).

\section{Acknowledgements}

We thank Dr. Hiroki Kato and Dr. Keiji Masuda (Kyushu University) for supporting a generation of Mff-deficient cells and Dr. Mitsuhiro Nishigori (Fukuoka University) for supporting a virus infection experiment. We also thank all members of Professor Nomura's (Kyushu University and Kurume University), Professor Ishihara’s (Osaka
University), and Professor Ogawa's (Kyushu University) laboratories for helpful discussions. We also thank the Research Support Center, Graduate School of Medical Science, Kyushu University for technical support. This work was supported by AMEDCREST (to N.I., under Grant Numbers JP20gm1110006 and JP20gm0810009), MEXTSupported Program for the Strategic Research Foundation at Private Universities to Kurume University, by the Japanese Society for the Promotion of Science KAKENHI (to M.N., Grant Number 17K09885; to L.W., Grant Number 16K19557; to N.I., Grant Number 17H03677), and by Robert T. Huang Entrepreneurship Center of Kyushu University (QREC) Academic Challenge 2017 grants-in-aid for scientific research (to Y.H.). This paper is dedicated to the memory of Hidenori Otera, our dear friend and colleague, who passed away on 19th Oct 2019.

\section{Author contributions}

M.N., Y.H., and N.I. designed the fundamental plan. Y.H. performed experiments. L.W. and T.I. supported experiments. H.O. provided cell lines and plasmids for Mff. T.K. provided plasmids of MAVS and performed virus infection experiments. K.M. and Y.O supervised the experiments. N.I. and Y.H. analyzed the data and wrote the manuscript. N.I. and Y.H. are responsible for all the data in this manuscript.

\section{Competing interests}

The authors declare no competing interests.

\section{Additional information}

Supplementary information is available for this paper at https://doi.org/10.1038/s41467020-19287-7.

Correspondence and requests for materials should be addressed to N.I.

Peer review information Nature Communications thanks Gerald Shadel, Phillip West, and the other anonymous reviewer(s) for their contribution to the peer review of this work. Peer review reports are available.

Reprints and permission information is available at http://www.nature.com/reprints

Publisher's note Springer Nature remains neutral with regard to jurisdictional claims in published maps and institutional affiliations.

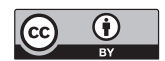

Open Access This article is licensed under a Creative Commons Attribution 4.0 International License, which permits use, sharing, adaptation, distribution and reproduction in any medium or format, as long as you give appropriate credit to the original author(s) and the source, provide a link to the Creative Commons license, and indicate if changes were made. The images or other third party material in this article are included in the article's Creative Commons license, unless indicated otherwise in a credit line to the material. If material is not included in the article's Creative Commons license and your intended use is not permitted by statutory regulation or exceeds the permitted use, you will need to obtain permission directly from the copyright holder. To view a copy of this license, visit http://creativecommons.org/ licenses/by/4.0/.

(C) The Author(s) 2020 